\title{
Extrapolation of the gold-catalyzed cycloisomerization to the palladium-catalyzed cross-coupling/cycloisomerization of acetylenic alcohols for the synthesis of polysubstituted furans: Scope and application to tandem processes
}

\author{
Chandrasekar Praveen a,*, Paramasivan T. Perumal ${ }^{b}$ \\ a Functional Materials Division, Central Electrochemical Research Institute (CSIR Laboratory), Karaikudi 630003, India \\ b Organic Chemistry Division, Central Leather Research Institute (CSIR Laboratory), Adyar, Chennai 600020, India
}

\section{A R T I C L E I N F O}

Article history:

Received 9 September 2015

Accepted 12 October 2015

Published 5 February 2016

Keywords:

Furan derivatives

Cycloisomerization

Gold catalysis

Palladium catalysis

One-pot operation

Tandem reactions

\begin{abstract}
A B S T R A C T
This paper describes the development of an integrated approach for the preparation of diverse furan derivatives from acetylenic alcohols by gold and palladium catalyzed $\pi$-activation chemistry. Notably, this new method was found to be amenable to cyclooctyl-containing substrates, which represents a significant extension to this methodology compared with our previous reports. Furthermore, this newly developed method allowed for the direct construction of cyclooctyl furans from their synthetic precursors under Sonogashira conditions. Experimental results revealed that palladium played two major functions in these reactions, including (1) an essential catalyst in the cross-coupling reaction of the substrates; and (2) facilitating the cyclization of the acetylenic alcohol intermediates through a typical $\pi$-activation process. The scope of this chemistry was highlighted by the one-pot synthesis of 3-iodofuran, which provided an opportunity for further functionalization (via coupling methods). Finally, the $\mathrm{AuBr}_{3}$ protocol was also elaborated to domino cyclization/C-H activation reactions, as well as the cyclization of acyclic precursors. Taken together, the results of this study demonstrate that gold and palladium catalysts can be used to complement each other in cyclization reactions.
\end{abstract}

(c) 2016, Dalian Institute of Chemical Physics, Chinese Academy of Sciences. Published by Elsevier B.V. All rights reserved.

\section{Introduction}

Homogeneous gold catalysts continue to attract increasing levels of interest in organic chemistry because of the efficiency, mild nature and unique properties [1-15]. Our collective understanding of gold catalysis has been facilitated by the theories of frontier molecular orbitals [16], relativistic effects $[17,18]$ and $\pi$-acidity $[19]$. The unusual reactivity of gold catalysts, namely the activation of $\mathrm{C}-\mathrm{C}$ triple bonds, has led to the development of a wide range of powerful methods for the effi- cient construction of diverse structural frameworks and molecular architectures [20,21]. The strong $\sigma$-donor and weak $\pi$-acceptor properties of alkynes render gold-complexed alkynes electrophilic and therefore opens the door for the discovery of new reactions [22,23]. In contrast to the vast majority of transition metal-catalyzed processes, the high oxidation potential of $\mathrm{Au}(\mathrm{I})$ to $\mathrm{Au}(\mathrm{III})$ allows most $\mathrm{Au}(\mathrm{I})$-catalyzed reactions to proceed without the requirement for an inert atmosphere. We have initiated a program to exploit the unique reactivity of alkyne precursors towards gold catalysis for the synthesis of

\footnotetext{
* Corresponding author. Tel: +91-9677733808; E-mail: chandrasekar.praveen@gmail.com This work was supported by the Department of Science and Technology (INSPIRE Faculty Program), India. 
carbo- and heterocyclic compounds [24-30]. Furans fused to cycloalkyl ring systems (e.g., cyclopentyl, cyclohexyl, cycloheptyl and cyclooctyl rings) have found numerous applications in a variety of different areas ranging from natural products to medicinal chemistry [31-38]. Consequently, contemporary synthetic chemistry has witnessed the development of a plethora of highly efficient synthetic strategies for the construction of these sub-structures [39-56]. However, despite significant advances in this area, there are still some challenging substrates for this reaction, including cyclooctyl derivatives. As part of our ongoing interest in the development of transition-metal catalysts for the synthesis of carbo- and heterocyclic systems [24-30,57-63], we recently reported the gold-catalyzed preparation of fused-furans containing five, six and seven member rings [24]. In a continuation of this work, it was envisaged that furans fused to an eight member ring could also be prepared in a similar manner. During our study towards the preparation of the requisite synthons, we found that the exposure of 2-bromocyclooct-2-enol to terminal acetylenes under Sonogashira conditions led to a sequential cross-coupling/ cycloisomerization process, which resulted in the formation of the fused furans. In other words, this process provided direct access to the desired cyclooctyl furans through a "gold-free" protocol. In this paper, we provide a detailed account of our work, including the synthetic elaboration of commercially available starting materials to give complex cyclooctyl furans, as well as the exploitation of tandem processes and the synthetic potential of these fused furan systems.

\section{Experimental}

\subsection{Materials, methods and instruments}

All of the commercially available solvents and reagents were used as supplied without further purification. Solutions in organic solvents were dried over anhydrous $\mathrm{Na}_{2} \mathrm{SO}_{4}$. Solvents were evaporated under reduced pressure. Melting points (MP) were obtained using open capillaries and have been reported as the uncorrected values. Infrared (IR) spectra were recorded on a Perkin-Elmer FTIR spectrophotometer (Hopkinton, MA, USA). The IR spectra were recorded as $\mathrm{KBr}$ pellets for solid compounds and neat sample for liquid compounds. ${ }^{1} \mathrm{H}$ and ${ }^{13} \mathrm{C}$ NMR spectra were recorded on a JEOL spectrometer (Peabody, MA, USA) at 500 and $125 \mathrm{MHz}$, respectively. The samples for NMR spectroscopy were prepared in DMSO- $d_{6}$ and $\mathrm{CDCl}_{3}$. The chemical shifts $(\delta)$ for the ${ }^{1} \mathrm{H}$ NMR spectra have been reported relative to tetramethylsilane (TMS, $\delta=0.00$ ), which was used as an internal reference standard, and expressed in parts per million (ppm). The number of protons $(n)$ for a given resonance has been indicated as $n \mathrm{H}$ and the spin multiplicities have been given as follows: s (singlet), d (doublet), t (triplet), dd (doublet of doublets), dt (doublet of triplets), ddd (doublet of doublet of doublets) and $m$ (multiplet). The coupling constants $($ ) have been given in hertz. GC-MS spectra were recorded on a Perkin-Elmer system. Elemental analyses were recorded on a Thermo Finnigan FLASH EA 1112CHN analyzer (Niulab Equipments, Mumbai, India). All of the compounds analyzed in the current study gave $\mathrm{C}, \mathrm{H}$ and $\mathrm{N}$ results that were within $\pm 0.5 \%$ of the theoretical values. Purifications by column chromatography were conducted over silica gel (100-200 mesh, SRL, Mumbai, India) using a mixture of petroleum ether and ethyl acetate (EtOAc) as the eluent. Analytical thin-layer chromatography (TLC) was performed on pre-coated plastic sheets of silica gel G/UV-254 of $0.2 \mathrm{~mm}$ in thickness (Macherey-Nagel, Düren, Germany) using analytical grade solvents. The TLC plates were visualized with iodine spray $\left(10 \% w / w \mathrm{I}_{2}\right.$ in silica gel), UV light $(\lambda=254$ and $365 \mathrm{~nm})$ and alkaline $\mathrm{KMnO}_{4}$ solution.

\subsection{General procedure for the synthesis of the 2-alkynyl-cycloalk-2-enols (5a-5I)}

Dry triethylamine (5 mL) was added to a mixture of iodo compound 3 (1.0 mmol), $\mathrm{Pd}\left(\mathrm{PPh}_{3}\right)_{2} \mathrm{Cl}_{2}(5 \mathrm{~mol} \%)$ and $\mathrm{CuI}(5$ mol\%) in an oven-dried flask under $\mathrm{N}_{2}$, and the resulting suspension was magnetically stirred for $5 \mathrm{~min}$. Terminal alkyne 4 (1.2 mmol) was then added to the reaction in a drop-wise manner, and the resulting mixture was stirred at room temperature until TLC analysis showed the disappearance of the 2-iodo-cycloalk-2-enol starting material $(\sim 6 \mathrm{~h})$. The reaction mixture was diluted with water $(50 \mathrm{~mL})$ and extracted with ethyl acetate $(4 \times 15 \mathrm{~mL})$. The combined organic extracts were dried over anhydrous $\mathrm{Na}_{2} \mathrm{SO}_{4}$ and the solvent was removed under reduced pressure to give the crude product as a residue. The residue was subsequently purified by column chromatography over silica gel eluting with a 9:1 $(v / v)$ mixture of EtOAc and petroleum ether to afford the pure product (5a-5l).

2-(Phenylethynyl)-cyclohept-2-enol (5a). Brown oil; IR $\left(\mathrm{CH}_{2} \mathrm{Cl}_{2}\right): 3395,2923,2354,1595,1438,752 \mathrm{~cm}^{-1} .{ }^{1} \mathrm{H}$ NMR (500 MHz, $\left.\mathrm{CDCl}_{3}\right): \delta_{\mathrm{H}} 1.67-1.71(\mathrm{~m}, 4 \mathrm{H}), 1.93-1.97(\mathrm{~m}, 2 \mathrm{H})$, 2.13-2.20 (m, 1H), 2.30-2.40 (m, 2H), $6.43(\mathrm{t}, 1 \mathrm{H}, J=6.8 \mathrm{~Hz})$, 7.29-7.30 (m, 4H), 7.42-7.44 (m, 2H). ${ }^{13} \mathrm{C}$ NMR (125 MHz, $\left.\mathrm{CDCl}_{3}\right): \delta \mathrm{c} 25.7,27.2,28.6,35.1,71.3,87.6,91.2,123.1,128.3$, 128.4, 131.2, 131.5, 138.9. GC-MS: $m / z=212$. Anal. Calcd. for $\mathrm{C}_{15} \mathrm{H}_{16} \mathrm{O}$ : C, 84.87\%; H, 7.60\%. Found: C, 84.80\%; H, 7.64\%.

2-(4-Pentylphenylethynyl)-cyclohept-2-enol (5b). Brown oil; IR $\left(\mathrm{CH}_{2} \mathrm{Cl}_{2}\right): 3435,2927,1679,1595,1438,838 \mathrm{~cm}^{-1} .{ }^{1} \mathrm{H}$ NMR (500 MHz, CDCl 3 ): $\delta_{\mathrm{H}} 0.87(\mathrm{t}, 3 \mathrm{H}, J=6.9 \mathrm{~Hz}), 1.28-1.32(\mathrm{~m}$, 5H), 1.51-1.68 (m, 5H), 1.93-1.95 (m, 2H), 2.15-2.18 (m, 2H), 2.25-2.30 (m, 1H), $2.58(\mathrm{t}, 2 \mathrm{H}, J=8.4 \mathrm{~Hz}), 4.43(\mathrm{brs}, 1 \mathrm{H}), 6.40(\mathrm{t}$, $1 \mathrm{H}, J=6.1 \mathrm{~Hz}), 7.10(\mathrm{~d}, 2 \mathrm{H}, J=7.6 \mathrm{~Hz}), 7.34(\mathrm{~d}, 2 \mathrm{H}, J=8.4 \mathrm{~Hz})$. ${ }^{13} \mathrm{C}$ NMR $\left(125 \mathrm{MHz}, \mathrm{CDCl}_{3}\right): \delta_{\mathrm{c}} 14.1,22.6,26.0,27.3,28.5,31.0$, 31.5, 35.1, 35.9, 71.3, 86.8, 91.6, 120.1, 123.2, 128.6, 131.4, 138.4, 143.5. GC-MS: $m / z=282$. Anal. Calcd. for $\mathrm{C}_{20} \mathrm{H}_{26} \mathrm{O}: \mathrm{C}$, 85.06\%; H, 9.28\%. Found: C, 84.98\%; H, 9.30\%.

2-Thiophen-2-ylethynyl-cyclohept-2-enol (5c). Brown oil; IR $\left(\mathrm{CH}_{2} \mathrm{Cl}_{2}\right): 3421,2925,2842,1654,1444,1079,850,698$ $\mathrm{cm}^{-1} .{ }^{1} \mathrm{H}$ NMR $\left(500 \mathrm{MHz}, \mathrm{CDCl}_{3}\right): \delta_{\mathrm{H}} 1.35-1.40(\mathrm{~m}, 1 \mathrm{H})$, 1.58-1.72 (m, 3H), 1.77-1.83 (m, 1H), 1.90-1.98 (m, 2H), $2.28-2.40(\mathrm{~m}, 2 \mathrm{H}), 4.43$ (brs, $1 \mathrm{H}), 6.41(\mathrm{t}, 1 \mathrm{H}, J=1.5 \mathrm{~Hz}), 6.94(\mathrm{t}$, $1 \mathrm{H}, J=5.3 \mathrm{~Hz}), 7.16(\mathrm{~d}, 1 \mathrm{H}, J=3.8 \mathrm{~Hz}), 7.23(\mathrm{~d}, 1 \mathrm{H}, J=5.3 \mathrm{~Hz})$. ${ }^{13} \mathrm{C}$ NMR $\left(125 \mathrm{MHz} \mathrm{CDCl}_{3}\right): \delta_{\mathrm{C}} 25.7,27.1,28.6,35.0,71.4,84.0$, 91.6, 123.2, 127.1, 127.2, 131.0, 131.8, 139.4. GC-MS: $\mathrm{m} / \mathrm{z}=$ 218. Anal. Calcd. for $\mathrm{C}_{13} \mathrm{H}_{14} \mathrm{OS}$ : C, 71.52\%; H, 6.46\%. Found: C, 
$71.40 \% ; \mathrm{H}, 6.50 \%$.

2-Hex-1-ynyl-cyclohept-2-enol (5d). Colorless oil; IR $\left(\mathrm{CH}_{2} \mathrm{Cl}_{2}\right)$ : 3368, 2928, 2851, 1707, 1447, 1065, 909, $732 \mathrm{~cm}^{-1}$. ${ }^{1} \mathrm{H}$ NMR (500 MHz, $\left.\mathrm{CDCl}_{3}\right): \delta_{\mathrm{H}} 0.96(\mathrm{t}, 3 \mathrm{H}, J=6.8 \mathrm{~Hz}), 1.28-1.34$ (m, 6H), 1.36-1.48 (m, 4H), 1.97-2.10 (m, 4H), $2.50(\mathrm{~m}, 1 \mathrm{H})$, 4.17 (brs, 1H), 6.07 (t, $1 \mathrm{H}, J=3.8 \mathrm{~Hz}) .{ }^{13} \mathrm{C}$ NMR $(125 \mathrm{MHz}$, $\left.\mathrm{CDCl}_{3}\right): \delta_{\mathrm{C}} 13.5,18.3,19.1,22.5,24.7,25.8,29.8,30.1,69.4,80.0$, 92.0, 130.2, 137.5. GC-MS: $m / z=192$. Anal. Calcd. for $\mathrm{C}_{13} \mathrm{H}_{20} \mathrm{O}$ : C, $81.20 \%$; H, 10.48\%. Found: C, 81.31\%; H, 10.54\%.

2-(1-Hydroxy-cyclohexylethynyl)-cyclohept-2-enol (5e). Colorless oil; IR $\left(\mathrm{CH}_{2} \mathrm{Cl}_{2}\right): 3500,3450,2930,1640,1435,1199$, $799 \mathrm{~cm}^{-1}$. ${ }^{1} \mathrm{H}$ NMR $\left(500 \mathrm{MHz}, \mathrm{CDCl}_{3}\right): \delta_{\mathrm{H}} 1.12-1.18(\mathrm{~m}, 4 \mathrm{H})$, $1.35-1.51(\mathrm{~m}, 6 \mathrm{H}), 1.59-1.65(\mathrm{~m}, 4 \mathrm{H}), 1.69-1.77(\mathrm{~m}, 4 \mathrm{H})$, 1.91-1.99 (m, 1H), 3.30 (brs, 1H), 4.20 (brs, $1 \mathrm{H}), 6.16(\mathrm{t}, 1 \mathrm{H}, J=$ $3.9 \mathrm{~Hz}) .{ }^{13} \mathrm{C}$ NMR $\left(125 \mathrm{MHz}, \mathrm{CDCl}_{3}\right): \delta \mathrm{c} 14.1,18.2,22.6,24.6$, 25.2, 25.6, 30.5, 39.0, 67.3, 69.4, 75.1, 82.9, 93.0, 124.4, 139.0. GC-MS: $m / z=234$. Anal. Calcd. for $\mathrm{C}_{15} \mathrm{H}_{22} \mathrm{O}_{2}$ : C, 76.88\%; $\mathrm{H}$, 9.46\%. Found: C, 77.00\%; H, 9.44\%.

2-(Phenylethynyl)-cyclohex-2-enol (5f). Brown oil; IR $\left(\mathrm{CH}_{2} \mathrm{Cl}_{2}\right): 3383,2928,1592,1053,755 \mathrm{~cm}^{-1} .{ }^{1} \mathrm{H} \mathrm{NMR}(500 \mathrm{MHz}$, $\left.\mathrm{CDCl}_{3}\right): \delta_{\mathrm{H}} 1.60-1.62(\mathrm{~m}, 1 \mathrm{H}), 1.75-1.78(\mathrm{~m}, 3 \mathrm{H}), 1.87-1.90(\mathrm{~m}$, $1 \mathrm{H}), 2.12-2.21(\mathrm{~m}, 2 \mathrm{H}), 4.26$ (brs, $1 \mathrm{H}), 6.30(\mathrm{t}, 1 \mathrm{H}, J=4.6 \mathrm{~Hz}$ ), 7.28-7.29 (m, 3H), 7.43-7.44 (m, 2H). ${ }^{13} \mathrm{C}$ NMR $(125 \mathrm{MHz}$, $\left.\mathrm{CDCl}_{3}\right): \delta_{\mathrm{C}} 18.1,26.0,30.6,66.9,88.4,89.3,123.2,124.2,128.2$, 128.3, 131.6, 137.8. GC-MS: $m / z=198$. Anal. Calcd. for $\mathrm{C}_{14} \mathrm{H}_{14} \mathrm{O}$ : C, 84.81\%; H, 7.12\%. Found: C, 84.91\%; H, 7.09\%.

2-(4-Pentylphenylethynyl)-cyclohex-2-enol (5g). Brown oil; IR $\left(\mathrm{CH}_{2} \mathrm{Cl}_{2}\right): 3400,2928,2363,1509,1461,1047,980 \mathrm{~cm}^{-1} .{ }^{1} \mathrm{H}$ NMR (500 MHz, $\left.\mathrm{CDCl}_{3}\right): \delta_{\mathrm{H}} 0.87(\mathrm{t}, 3 \mathrm{H}, J=6.9 \mathrm{~Hz}), 1.25-1.32(\mathrm{~m}$, $4 \mathrm{H}), 1.57-1.60(\mathrm{~m}, 3 \mathrm{H}), 1.74-1.76(\mathrm{~m}, 2 \mathrm{H}), 1.88-1.90(\mathrm{~m}, 1 \mathrm{H})$, 2.11-2.22 (m, 3H), $2.57(\mathrm{t}, 2 \mathrm{H}, J=7.6 \mathrm{~Hz}), 4.22(\mathrm{brs}, 1 \mathrm{H}), 6.28(\mathrm{t}$, $1 \mathrm{H}, J=4.6 \mathrm{~Hz}), 7.11(\mathrm{~d}, 2 \mathrm{H}, J=8.4 \mathrm{~Hz}), 7.34(\mathrm{~d}, 2 \mathrm{H}, J=8.4 \mathrm{~Hz})$. ${ }^{13} \mathrm{C}$ NMR $\left(125 \mathrm{MHz}, \mathrm{CDCl}_{3}\right): \delta \mathrm{c} 14.1,18.2,22.6,26.0,30.6,31.0$, 31.5, 35.9, 67.0, 87.5, 89.6, 120.2, 124.4, 128.5, 131.5, 137.3, 143.4. GC-MS: $m / z=268$. Anal. Calcd. for $\mathrm{C}_{19} \mathrm{H}_{24} \mathrm{O}: \mathrm{C}, 85.03 \%$; $\mathrm{H}$, 9.01\%. Found: C, 85.18\%; H, 8.96\%.

2-Pyridin-2-ylethynyl-cyclohex-2-enol (5h). Yellow oil; IR $\left(\mathrm{CH}_{2} \mathrm{Cl}_{2}\right): 3450,3398,2929,2369,1500,1450,1055,977 \mathrm{~cm}^{-1}$. ${ }^{1} \mathrm{H}$ NMR (500 MHz, $\left.\mathrm{CDCl}_{3}\right): \delta_{\mathrm{H}} 1.58-1.60(\mathrm{~m}, 1 \mathrm{H}), 1.75-1.77(\mathrm{~m}$, $2 \mathrm{H}), 1.86-1.88(\mathrm{~m}, 1 \mathrm{H}), 2.12-2.18(\mathrm{~m}, 2 \mathrm{H}), 2.21-2.24(\mathrm{~m}, 1 \mathrm{H})$, 4.31 (brs, $1 \mathrm{H}), 6.41(\mathrm{t}, 1 \mathrm{H}, J=4.6 \mathrm{~Hz}), 7.17(\mathrm{t}, 1 \mathrm{H}, J=7.6 \mathrm{~Hz})$, $7.39(\mathrm{~d}, 1 \mathrm{H}, J=6.8 \mathrm{~Hz}), 7.61(\mathrm{t}, 1 \mathrm{H}, J=7.6 \mathrm{~Hz}), 8.53(\mathrm{~d}, 1 \mathrm{H}, J=$ $5.3 \mathrm{~Hz}) .{ }^{13} \mathrm{C}$ NMR $\left(125 \mathrm{MHz}, \mathrm{CDCl}_{3}\right): \delta_{\mathrm{c}} 18.1,26.2,30.7,66.5$, 88.3, 89.1, 122.6, 124.1, 127.0, 136.2, 139.6, 143.4, 149.8. GC-MS: $m / z=199$. Anal. Calcd. for $\mathrm{C}_{13} \mathrm{H}_{13} \mathrm{NO}$ : C, 78.36\%; $\mathrm{H}$, 6.58\%; N, 7.03\%. Found: C, 78.47\%; H, 6.56\%; N, 7.00\%.

2-Hex-1-ynyl-cyclohex-2-enol (5i). Colorless oil; IR $\left(\mathrm{CH}_{2} \mathrm{Cl}_{2}\right)$ : 3430, 2930, 1501, 1455, 990, $803 \mathrm{~cm}^{-1}$. ${ }^{1} \mathrm{H}$ NMR (500 MHz, $\left.\mathrm{CDCl}_{3}\right): \delta_{\mathrm{H}} 0.96(\mathrm{t}, 3 \mathrm{H}, J=6.8 \mathrm{~Hz}), 1.32-1.44(\mathrm{~m}, 4 \mathrm{H}), 1.60-1.79$ (m, 4H), 1.90-2.15 (m, 5H), 4.09 (brs, 1H), 5.91 (t, 1H, $J=4.3$ $\mathrm{Hz}$ ). ${ }^{13} \mathrm{C}$ NMR $\left(125 \mathrm{MHz}, \mathrm{CDCl}_{3}\right): \delta \mathrm{c} 13.6,19.2,21.5,25.0,28.4$, 31.2, 36.8, 69.9, 78.7, 90.3, 132.3, 137.8. GC-MS: $m / z=178$. Anal. Calcd. for $\mathrm{C}_{12} \mathrm{H}_{18} \mathrm{O}$ : C, 80.85\%; H, 10.18\%. Found: C, 80.99\%; H, 10.12\%.

2-(1-Hydroxy-cyclohexylethynyl)-cyclohex-2-enol (5j). Colorless oil; IR $\left(\mathrm{CH}_{2} \mathrm{Cl}_{2}\right)$ : 3505, 3451, 2929, 1513, 1219, $782 \mathrm{~cm}^{-1}$. ${ }^{1} \mathrm{H}$ NMR (500 MHz, $\left.\mathrm{CDCl}_{3}\right): \delta_{\mathrm{H}} 1.18-1.23(\mathrm{~m}, 2 \mathrm{H}), 1.43-1.59(\mathrm{~m}$,
$6 \mathrm{H}), 1.63-1.73(\mathrm{~m}, 3 \mathrm{H}), 1.79-1.91(\mathrm{~m}, 3 \mathrm{H}), 2.00-2.05(\mathrm{~m}, 1 \mathrm{H})$, 2.08-2.15 (m, 1H), 2.80-2.99 (m, 1H), 3.35 (brs, 1H), 4.14 (brs, $1 \mathrm{H}), 6.15(\mathrm{t}, 1 \mathrm{H}, J=3.8 \mathrm{~Hz}) .{ }^{13} \mathrm{C}$ NMR $\left(125 \mathrm{MHz}, \mathrm{CDCl}_{3}\right): \delta \mathrm{c} 18.3$, 23.5, 25.2, 25.7, 25.8, 30.7, 40.0, 68.9, 83.3, 92.9, 123.9, 129.9. GC-MS: $m / z=220$. Anal. Calcd. for $\mathrm{C}_{14} \mathrm{H}_{20} \mathrm{O}_{2}$ : C, 76.33\%; $\mathrm{H}$, 9.15\%. Found: C, 76.44\%; H, 9.12\%.

2-(Phenylethynyl)-cyclopent-2-enol (5k). Brown oil; IR $\left(\mathrm{CH}_{2} \mathrm{Cl}_{2}\right): 3368,2924,2363,1485,1309,1048,756 \mathrm{~cm}^{-1} \cdot{ }^{1} \mathrm{H}$ NMR (500 MHz, $\left.\mathrm{CDCl}_{3}\right): \delta_{\mathrm{H}} 1.82-1.84(\mathrm{~m}, 1 \mathrm{H}), 2.14(\mathrm{~s}, 1 \mathrm{H})$, 2.34-2.37 (m, 2H), 2.58-2.60 (m, 1H), 4.87 (brs, 1H), $6.29(\mathrm{t}$, $1 \mathrm{H}, J=3.05 \mathrm{~Hz}), 7.29-7.31(\mathrm{~m}, 2 \mathrm{H}), 7.45-7.46(\mathrm{~m}, 3 \mathrm{H}) .{ }^{13} \mathrm{C} \mathrm{NMR}$ (125 MHz, $\left.\mathrm{CDCl}_{3}\right): \delta \mathrm{c} 31.0,32.9,77.1,84.7,92.1,123.1,128.2$, 128.4 (2C), 131.7, 140.5. GC-MS: $m / z=184$. Anal. Calcd. for $\mathrm{C}_{13} \mathrm{H}_{12} \mathrm{O}$ : C, 84.75\%; H, 6.57\%. Found: C, 84.80\%; H, 6.55\%.

2-(4-Pentylphenylethynyl)-cyclopent-2-enol (5l). Brown oil; IR $\left(\mathrm{CH}_{2} \mathrm{Cl}_{2}\right): 3377,2914,2363,1491,1313,1041,759 \mathrm{~cm}^{-1} .{ }^{1} \mathrm{H}$ NMR (500 MHz, CDCl 3$): \delta_{\mathrm{H}} 0.87(\mathrm{t}, 3 \mathrm{H}, J=6.9 \mathrm{~Hz}), 1.27-1.34(\mathrm{~m}$, $4 \mathrm{H}), 1.58-1.63(\mathrm{~m}, 2 \mathrm{H}), 1.80-1.84(\mathrm{~m}, 1 \mathrm{H}), 2.03(\mathrm{~s}, 1 \mathrm{H})$, 2.33-2.37 (m, 2H), 2.56-2.62 (m, 3H), $4.86(\mathrm{~s}, 1 \mathrm{H}), 6.26(\mathrm{~s}, 1 \mathrm{H})$, $7.11(\mathrm{~d}, 2 \mathrm{H}, J=7.6 \mathrm{~Hz}), 7.36$ (d, 2H, $J=8.4 \mathrm{~Hz}) .{ }^{13} \mathrm{C}$ NMR $(125$ $\left.\mathrm{MHz}, \mathrm{CDCl}_{3}\right): \delta_{\mathrm{c}} 14.1,22.6,30.9,31.0,31.5,32.9,35.9,79.1,83.9$, $92.5,120.2,128.4,128.5,131.6,139.9,143.5$. GC-MS: $m / z=$ 254. Anal. Calcd. for $\mathrm{C}_{18} \mathrm{H}_{22} \mathrm{O}$ : C, 84.99\%; $\mathrm{H}, 8.72 \%$. Found: C, 84.85\%; H, 8.78\%.

Experimental procedure for the synthesis of compound (5m). A mixture of compound 3a (205 mg, $1 \mathrm{mmol}$ ), phenylethynylcopper(I) (164 mg, $1 \mathrm{mmol}$ ) and dry Et3 $\mathrm{N}$ (5 mL) was heated at $80{ }^{\circ} \mathrm{C}$ under an atmosphere of $\mathrm{N}_{2}$ for $6 \mathrm{~h}$. The mixture was then poured into ice-cold water $(25 \mathrm{~mL})$ and extracted with $\mathrm{Et}_{2} \mathrm{O}(3 \times 10 \mathrm{~mL})$. The combined organic extracts were washed sequentially with $2 \mathrm{M} \mathrm{HCl}(5 \mathrm{~mL}), 5 \% \mathrm{NaHCO}_{3}(5$ $\mathrm{mL})$ and water $(10 \mathrm{~mL})$, and then dried over anhydrous $\mathrm{MgSO}_{4}$. The solvent was removed under reduced pressure to afford the crude product, which was purified by column chromatography over silica gel eluting with a 1:4 $(\mathrm{v} / \mathrm{v})$ mixture of EtOAc and petroleum ether to afford the pure product $\mathbf{5 m}$ as a light yellow solid. IR (KBr): 3390, 2921, 2352, 1595, 1431, $748 \mathrm{~cm}^{-1} .{ }^{1} \mathrm{H}$ NMR (500 MHz, $\left.\mathrm{CDCl}_{3}\right): \delta_{\mathrm{H}} 1.62-1.77(\mathrm{~m}, 6 \mathrm{H}), 1.99-2.08(\mathrm{~m}$, $2 \mathrm{H}), 2.17-2.23(\mathrm{~m}, 1 \mathrm{H}), 2.34-2.43(\mathrm{~m}, 2 \mathrm{H}), 6.45(\mathrm{t}, 1 \mathrm{H}, J=6.9$ $\mathrm{Hz}), 7.32-7.40(\mathrm{~m}, 4 \mathrm{H}), 7.48-7.52(\mathrm{~m}, 2 \mathrm{H}) .{ }^{13} \mathrm{C} \mathrm{NMR}(125 \mathrm{MHz}$, $\left.\mathrm{CDCl}_{3}\right): \delta_{\mathrm{C}} 24.3,25.9,27.5,28.9,35.4,71.7,87.9,91.6,123.6$, 128.5, 128.9, 131.7, 131.9, 140.4. GC-MS: $m / z=226$. Anal. Calcd. for $\mathrm{C}_{16} \mathrm{H}_{18} \mathrm{O}$ : C, 84.80\%; H, 8.02\%. Found: $\mathrm{C}, 84.95 \%$; $\mathrm{H}$, $7.96 \%$.

\subsection{General procedure for the synthesis of fused furans (6a-6l and $6 q)$}

$\mathrm{AuBr}_{3}(5 \mathrm{~mol} \%)$ was added a stirred solution of 2-acetylenic alcohol 5 (1.0 mmol) in dichloroethane $(1 \mathrm{~mL})$ under $\mathrm{N}_{2}$, and the resulting mixture was stirred at the specified temperature and the specified time (see Table 2 and Schemes 2 and 7). Upon completion of the reaction, as indicated by TLC, the reaction mixture was quenched with $10 \mathrm{~mL}$ of water and extracted with dichloromethane $(3 \times 5 \mathrm{~mL})$. The combined organic extracts were dried over anhydrous $\mathrm{Na}_{2} \mathrm{SO}_{4}$, filtered and concentrated under reduced pressure to afford the crude product, which was 
purified by column chromatography over silica gel (100-200 mesh) eluting with a mixture of cyclohexane and EtOAc to afford pure product (6a-6l and $\mathbf{6 q}$ ).

2-Phenyl-5,6,7,8-tetrahydro-4H-cyclohepta[b]furan (6a). Colorless oil; IR $\left(\mathrm{CH}_{2} \mathrm{Cl}_{2}\right): 2924,2851,1676,1450,803 \mathrm{~cm}^{-1} .{ }^{1} \mathrm{H}$ NMR (500 MHz, $\left.\mathrm{CDCl}_{3}\right): \delta_{\mathrm{H}} 1.75-1.82(\mathrm{~m}, 6 \mathrm{H}), 2.53(\mathrm{t}, 2 \mathrm{H}, J=5.3$ $\mathrm{Hz}), 2.86(\mathrm{t}, 2 \mathrm{H}, J=6.1 \mathrm{~Hz}), 6.45(\mathrm{~s}, 1 \mathrm{H}), 7.21(\mathrm{t}, 1 \mathrm{H}, J=7.6 \mathrm{~Hz})$, $7.36(\mathrm{t}, 2 \mathrm{H}, J=8.4 \mathrm{~Hz}), 7.63(\mathrm{~d}, 2 \mathrm{H}, J=7.6 \mathrm{~Hz}) .{ }^{13} \mathrm{C}$ NMR $(125$ $\mathrm{MHz}, \mathrm{CDCl}_{3}$ ): $\delta \mathrm{c} 26.3,26.7,28.8,29.1,30.9,108.8,123.0,123.2$, 126.5, 128.6, 131.4, 149.8, 153.4. GC-MS: $m / z=212$. Anal. Calcd. for $\mathrm{C}_{15} \mathrm{H}_{16} \mathrm{O}$ : C, $84.87 \%$; $\mathrm{H}, 7.60 \%$. Found: $\mathrm{C}, 85.00 \%$; $\mathrm{H}$, $7.55 \%$.

2-(4-Pentylphenyl)-5,6,7,8-tetrahydro-4H-cyclohepta[b]furan (6b). Colorless liquid; IR $\left(\mathrm{CH}_{2} \mathrm{Cl}_{2}\right): 2926,2851,1676,1450,803$ $\mathrm{cm}^{-1} .{ }^{1} \mathrm{H}$ NMR $\left(500 \mathrm{MHz}, \mathrm{CDCl}_{3}\right): \delta_{\mathrm{H}} 0.90(\mathrm{t}, 3 \mathrm{H}, J=6.9 \mathrm{~Hz})$, $1.33-1.36(\mathrm{~m}, 5 \mathrm{H}), 1.62(\mathrm{t}, 2 \mathrm{H}, J=7.6 \mathrm{~Hz}), 1.72-1.79(\mathrm{~m}, 5 \mathrm{H})$, $2.51(\mathrm{t}, 2 \mathrm{H}, J=6.1 \mathrm{~Hz}), 2.59(\mathrm{t}, 2 \mathrm{H}, J=7.6 \mathrm{~Hz}), 2.83(\mathrm{t}, 2 \mathrm{H}, J=6.1$ $\mathrm{Hz}), 6.37$ (s, 1H), 7.15 (d, $2 \mathrm{H}, J=8.4 \mathrm{~Hz}), 7.51$ (d, $2 \mathrm{H}, J=7.6 \mathrm{~Hz}$ ). ${ }^{13} \mathrm{C}$ NMR (125 MHz, $\mathrm{CDCl}_{3}$ ): $\delta_{\mathrm{C}} 14.1,22.6,26.3,26.7,28.8,29.1$, 30.9, 31.2, 31.6, 35.7, 108.0, 122.8, 123.2, 128.6, 128.9, 141.3, 150.1, 152.9. GC-MS: $m / z=282$. Anal. Calcd. for $\mathrm{C}_{20} \mathrm{H}_{26} \mathrm{O}: \mathrm{C}$, 85.06\%; H, 9.28\%. Found: C, 85.00\%; H, 9.30\%.

2-Thiophen-2-yl-5,6,7,8-tetrahydro-4H-cyclohepta[b]furan (6c). Brown oil; IR $\left(\mathrm{CH}_{2} \mathrm{Cl}_{2}\right)$ : 2921, 2847, 1663, 1573, 1443, 1218, 1080, 840, $691 \mathrm{~cm}^{-1} .{ }^{1} \mathrm{H}$ NMR $\left(500 \mathrm{MHz}, \mathrm{CDCl}_{3}\right): \delta_{\mathrm{H}}$ $1.72-1.73(\mathrm{~m}, 4 \mathrm{H}), 1.77-1.79(\mathrm{~m}, 2 \mathrm{H}), 2.49(\mathrm{t}, 2 \mathrm{H}, J=6.1 \mathrm{~Hz})$, $2.81(\mathrm{t}, 2 \mathrm{H}, J=6.1 \mathrm{~Hz}), 6.29(\mathrm{~s}, 1 \mathrm{H}), 7.00\left(\mathrm{dd}, 1 \mathrm{H}, J_{1}=3.8 \mathrm{~Hz}, J_{2}=\right.$ $3.8 \mathrm{~Hz}), 7.14-7.15(\mathrm{~m}, 2 \mathrm{H}) .{ }^{13} \mathrm{C}$ NMR $\left(125 \mathrm{MHz}, \mathrm{CDCl}_{3}\right): \delta_{\mathrm{C}} 26.2$, 26.6, 28.7, 29.0, 30.8, 108.8, 121.3, 122.9, 123.1, 127.6, 134.5, 145.5, 153.0. GC-MS: $m / z=218$. Anal. Calcd. for $\mathrm{C}_{13} \mathrm{H}_{14} \mathrm{OS}: \mathrm{C}$, $71.52 \%$; H, 6.46\%. Found: C, 71.58\%; H, 6.45\%.

2-Butyl-5,6,7,8-tetrahydro-4H-cyclohepta[b]furan (6d). Colorless liquid; IR $\left(\mathrm{CH}_{2} \mathrm{Cl}_{2}\right)$ : 2919, 2850, 1660, 1565, 1435, $1211,1075,850 \mathrm{~cm}^{-1} .{ }^{1} \mathrm{H}$ NMR $\left(500 \mathrm{MHz}, \mathrm{CDCl}_{3}\right): \delta_{\mathrm{H}} 0.95(\mathrm{t}, 3 \mathrm{H}$, $J=6.8 \mathrm{~Hz}), 1.27-1.34(\mathrm{~m}, 4 \mathrm{H}), 1.55-1.65(\mathrm{~m}, 6 \mathrm{H}), 2.31-2.55(\mathrm{~m}$, 6H), 6.03 (s, 1H). ${ }^{13} \mathrm{C}$ NMR (125 MHz, $\left.\mathrm{CDCl}_{3}\right): \delta_{\mathrm{c}} 14.0,20.2,20.3$, 24.4, 29.9, 30.1, 31.2, 32.6, 32.8, 106.4, 112.8, 149.8, 152.0. GC-MS: $m / z=192$. Anal. Calcd. for $\mathrm{C}_{13} \mathrm{H}_{20} \mathrm{O}: \mathrm{C}, 81.20 \%$; $\mathrm{H}$, $10.48 \%$. Found: C, $81.30 \%$; H, 10.44\%.

1-(5,6,7,8-Tetrahydro-4H-cyclohepta[b]furan-2-yl)-cyclohexanol (6e). Colorless liquid; IR $\left(\mathrm{CH}_{2} \mathrm{Cl}_{2}\right)$ : 3477, 2940, 2935, $1652,1439,1231,799,702 \mathrm{~cm}^{-1}$. ${ }^{1} \mathrm{H}$ NMR $\left(500 \mathrm{MHz}, \mathrm{CDCl}_{3}\right): \delta_{\mathrm{H}}$ $1.19-1.26(\mathrm{~m}, 2 \mathrm{H}), 1.55-1.63(\mathrm{~m}, 6 \mathrm{H}), 1.72-1.79(\mathrm{~m}, 4 \mathrm{H})$, 1.75-1.81 (m, 4H), 1.92-2.15 (m, 4H), 3.99 (brs, 1H), 6.49 (s, 1H). ${ }^{13} \mathrm{C}$ NMR (125 MHz, $\left.\mathrm{CDCl}_{3}\right): \delta \mathrm{c} 16.9,17.3,18.5,18.9,19.9$, 20.1, 22.8, 31.2, 35.3, 105.9, 117.8, 140.7, 151.0. GC-MS: $m / z=$ 234. Anal. Calcd. for $\mathrm{C}_{15} \mathrm{H}_{22} \mathrm{O}_{2}$ : C, 76.88\%; H, 9.46\%. Found: C, 77.01\%; H, 9.44\%.

2-Phenyl-4,5,6,7-tetrahydro-benzofuran (6f). Colorless liquid; IR $\left(\mathrm{CH}_{2} \mathrm{Cl}_{2}\right)$ : 2934, 2851, 1669, 1447, 1247, 760, $690 \mathrm{~cm}^{-1}$. ${ }^{1} \mathrm{H}$ NMR $\left(500 \mathrm{MHz}, \mathrm{CDCl}_{3}\right): \delta_{\mathrm{H}} 1.75-1.78(\mathrm{~m}, 2 \mathrm{H}), 1.84-1.89(\mathrm{~m}$, $2 \mathrm{H}), 2.46(\mathrm{t}, 2 \mathrm{H}, J=6.1 \mathrm{~Hz}), 2.67(\mathrm{t}, 2 \mathrm{H}, J=6.1 \mathrm{~Hz}), 6.47(\mathrm{~s}, 1 \mathrm{H})$, $7.20(\mathrm{t}, 1 \mathrm{H}, J=7.6 \mathrm{~Hz}), 7.35(\mathrm{t}, 2 \mathrm{H}, J=7.6 \mathrm{~Hz}), 7.62(\mathrm{~d}, 2 \mathrm{H}, J=6.9$ Hz). ${ }^{13} \mathrm{C}$ NMR $\left(125 \mathrm{MHz}, \mathrm{CDCl}_{3}\right): \delta \mathrm{c} 22.2,23.1,23.2,23.4,106.1$, 119.0, 123.3, 126.6, 128.6, 131.5, 150.9. GC-MS: $m / z=198$. Anal. Calcd. for $\mathrm{C}_{14} \mathrm{H}_{14} \mathrm{O}$ : C, 84.81\%; H, 7.12\%. Found: C, 84.75\%; H, 7.15\%.
2-(4-Pentylphenyl)-4,5,6,7-tetrahydro-benzofuran (6g). Colorless liquid; IR $\left(\mathrm{CH}_{2} \mathrm{Cl}_{2}\right): 2928,2851,2354,1495,1452$, 909, $838 \mathrm{~cm}^{-1} .{ }^{1} \mathrm{H}$ NMR $\left(500 \mathrm{MHz}, \mathrm{CDCl}_{3}\right): \delta_{\mathrm{H}} 0.92(\mathrm{t}, 3 \mathrm{H}, J=6.8$ $\mathrm{Hz}), 1.30-1.36(\mathrm{~m}, 5 \mathrm{H}), 1.64-1.66(\mathrm{~m}, 3 \mathrm{H}), 1.80-1.88(\mathrm{~m}, 2 \mathrm{H})$, 1.99-2.28 (m, 2H), 2.59-2.77 (m, 4H), $6.35(\mathrm{~s}, 1 \mathrm{H}), 7.18(\mathrm{~d}, 1 \mathrm{H}, J$ $=8.4 \mathrm{~Hz}), 7.25(\mathrm{~d}, 1 \mathrm{H}, J=7.6 \mathrm{~Hz}), 7.52-7.57(\mathrm{~m}, 2 \mathrm{H}) .{ }^{13} \mathrm{C} \mathrm{NMR}$ (125 MHz, $\mathrm{CDCl}_{3}$ ): $\delta_{\mathrm{c}} 14.2,22.1,22.9,23.1,23.5,30.7,31.2,31.6$, 35.8, 105.2, 118.8, 123.3, 126.6, 141.5, 147.7, 150.1, 152.1. GC-MS: $m / z=268$. Anal. Calcd. for $\mathrm{C}_{19} \mathrm{H}_{24} \mathrm{O}$ : C, 85.03\%; $\mathrm{H}$, 9.01\%. Found: C, $84.98 \% ; \mathrm{H}, 9.03 \%$.

2-(4,5,6,7-Tetrahydro-benzofuran-2-yl)-pyridine (6h). Yellow oil; IR $\left(\mathrm{CH}_{2} \mathrm{Cl}_{2}\right): 3400,2930,2850,1680,1450,823 \mathrm{~cm}^{-1} .{ }^{1} \mathrm{H}$ NMR $\left(500 \mathrm{MHz}, \mathrm{CDCl}_{3}\right): \delta_{\mathrm{H}} 1.65-1.74(\mathrm{~m}, 3 \mathrm{H}), 2.21-2.24(\mathrm{~m}$, $2 \mathrm{H}), 2.38-2.40(\mathrm{~m}, 1 \mathrm{H}), 2.44(\mathrm{t}, 1 \mathrm{H}, J=7.6 \mathrm{~Hz}), 2.66(\mathrm{t}, 1 \mathrm{H}, J=$ $6.1 \mathrm{~Hz}), 6.36(\mathrm{~s}, 1 \mathrm{H}), 7.14-7.17(\mathrm{~m}, 1 \mathrm{H}), 7.56(\mathrm{t}, 1 \mathrm{H}, J=7.6 \mathrm{~Hz})$, 7.63-7.65 (m, 1H), 8.57 (d, $1 \mathrm{H}, J=3.8 \mathrm{~Hz}) .{ }^{13} \mathrm{C}$ NMR $(125 \mathrm{MHz}$, $\mathrm{CDCl}_{3}$ ): $\delta_{\mathrm{C}} 19.7,24.6,27.7,29.7,82.9,104.2,113.7,120.1,123.0$, 136.4, 143.2, 149.6, 160.1. GC-MS: $m / z=199$. Anal. Calcd. for $\mathrm{C}_{13} \mathrm{H}_{13} \mathrm{NO}$ : C, 78.36\%; H, 6.58; N, 7.03\%. Found: C, 78.25\%; H, $6.60 \% ; \mathrm{N}, 7.07 \%$.

2-Butyl-4,5,6,7-tetrahydrobenzofuran (6i). Colorless oil; IR $\left(\mathrm{CH}_{2} \mathrm{Cl}_{2}\right): 2935,2853,1675,1447,799,763 \mathrm{~cm}^{-1} .{ }^{1} \mathrm{H}$ NMR $(500$ $\left.\mathrm{MHz}, \mathrm{CDCl}_{3}\right): \delta_{\mathrm{H}} 0.93(\mathrm{t}, 3 \mathrm{H}, J=6.9 \mathrm{~Hz}), 1.33-1.69(\mathrm{~m}, 8 \mathrm{H})$, 2.35-2.60 (m, 6H), $6.01(\mathrm{~s}, 1 \mathrm{H}) .{ }^{13} \mathrm{C}$ NMR $\left(125 \mathrm{MHz}, \mathrm{CDCl}_{3}\right): \delta \mathrm{c}$ 13.4, 20.9, 21.2, 29.5, 31.1, 32.3, 35.4, 36.0, 106.3, 112.6, 149.5, 151.1. GC-MS: $m / z=178$. Anal. Calcd. for $\mathrm{C}_{12} \mathrm{H}_{18} \mathrm{O}: \mathrm{C}, 80.85 \%$; $\mathrm{H}$, $10.18 \%$. Found: C, $80.74 \%$; H, $10.22 \%$.

1-(4,5,6,7-Tetrahydro-benzofuran-2-yl)-cyclohexanol (6j). Colorless oil; IR $\left(\mathrm{CH}_{2} \mathrm{Cl}_{2}\right)$ : 3480, 2935, 2929, 1669, 1447, 1250, $800,713 \mathrm{~cm}^{-1} .{ }^{1} \mathrm{H}$ NMR $\left(500 \mathrm{MHz}, \mathrm{CDCl}_{3}\right): \delta_{\mathrm{H}} 1.20-1.25(\mathrm{~m}, 2 \mathrm{H})$, $1.49-1.61(\mathrm{~m}, 6 \mathrm{H}), 1.65-1.69(\mathrm{~m}, 4 \mathrm{H}), 1.72-1.75(\mathrm{~m}, 2 \mathrm{H})$, 1.89-2.10 (m, 4H), 3.95 (brs, $1 \mathrm{H}), 6.51(\mathrm{~s}, 1 \mathrm{H}) .{ }^{13} \mathrm{C}$ NMR $(125$ $\mathrm{MHz}, \mathrm{CDCl}_{3}$ ): $\delta \mathrm{c} 17.2,17.4,18.0,18.2,19.0,20.1,22.6,35.7$, 105.2, 118.0, 141.9, 151.2. GC-MS: $m / z=220$. Anal. Calcd. for $\mathrm{C}_{14} \mathrm{H}_{20} \mathrm{O}_{2}$ : C, 76.33\%; H, 9.15\%. Found: C, 76.25\%; H, 9.17\%.

2-Phenyl-5,6-dihydro-4H-cyclopenta[b]furan (6k). Colorless oil; IR $\left(\mathrm{CH}_{2} \mathrm{Cl}_{2}\right): 2935,2859,1681,1453,810 \mathrm{~cm}^{-1} .{ }^{1} \mathrm{H}$ NMR ( $\left.500 \mathrm{MHz}, \mathrm{CDCl}_{3}\right): \delta_{\mathrm{H}} 1.79-1.83(\mathrm{~m}, 2 \mathrm{H}), 1.95(\mathrm{t}, 2 \mathrm{H}, J=6.3 \mathrm{~Hz})$, $2.56(\mathrm{t}, 2 \mathrm{H}, J=6.2 \mathrm{~Hz}), 6.50(\mathrm{~s}, 1 \mathrm{H}), 7.15(\mathrm{t}, 1 \mathrm{H}, J=7.6 \mathrm{~Hz}), 7.34$ $(\mathrm{t}, 2 \mathrm{H}, J=8.4 \mathrm{~Hz}), 7.63(\mathrm{~d}, 2 \mathrm{H}, J=7.6 \mathrm{~Hz}) .{ }^{13} \mathrm{C}$ NMR $(125 \mathrm{MHz}$, $\left.\mathrm{CDCl}_{3}\right): \delta_{\mathrm{C}} 23.5,23.7,24.2,105.1,118.0,122.3,125.5,127.6$, 130.5, 150.7, 151.9. GC-MS: $m / z=184$. Anal. Calcd. for $\mathrm{C}_{13} \mathrm{H}_{12} \mathrm{O}$ : C, 84.75\%; H, 6.57\%. Found: C, 84.86\%; H, 6.54\%.

2-(4-Pentylphenyl)-5,6-dihydro-4H-cyclopenta[b]furan (61). Colorless oil; IR $\left(\mathrm{CH}_{2} \mathrm{Cl}_{2}\right): 2931,2856,1680,1455,892,800$ $\mathrm{cm}^{-1} .{ }^{1} \mathrm{H}$ NMR $\left(500 \mathrm{MHz}, \mathrm{CDCl}_{3}\right): \delta_{\mathrm{H}} 0.94(\mathrm{t}, 3 \mathrm{H}, J=6.8 \mathrm{~Hz})$, 1.36-1.41 (m, 5H), 1.68-1.71 (m, 3H), 1.76-1.80 (m, 2H), 1.93 $(\mathrm{t}, 2 \mathrm{H}, J=6.2 \mathrm{~Hz}), 2.56(\mathrm{t}, 2 \mathrm{H}, J=6.3 \mathrm{~Hz}), 6.43(\mathrm{~s}, 1 \mathrm{H}), 7.22(\mathrm{~d}$, $2 \mathrm{H}, J=7.6 \mathrm{~Hz}), 7.34(\mathrm{~d}, 2 \mathrm{H}, J=7.6 \mathrm{~Hz}) .{ }^{13} \mathrm{C}$ NMR $(125 \mathrm{MHz}$, $\left.\mathrm{CDCl}_{3}\right): \delta_{\mathrm{c}} 14.3,22.0,22.8,23.0,30.6,31.1,31.6,35.8,104.9$, $117.9,123.4,123.8,128.7,148.0,150.0,152.0$. GC-MS: $m / z=$ 254. Anal. Calcd. for $\mathrm{C}_{18} \mathrm{H}_{22} \mathrm{O}: \mathrm{C}, 84.99 \%$; $\mathrm{H}, 8.72 \%$. Found: $\mathrm{C}$, $85.10 \% ; \mathrm{H}, 8.67 \%$.

2,3-Dimethylfuran (6q). Colorless liquid; IR (neat): 3150, $3115,1501,1164,1075,801 \mathrm{~cm}^{-1}$. ${ }^{1} \mathrm{H}$ NMR $\left(500 \mathrm{MHz}, \mathrm{CDCl}_{3}\right)$ : $\delta_{\mathrm{H}} 1.91(\mathrm{~s}, 3 \mathrm{H}), 2.29(\mathrm{~s}, 3 \mathrm{H}), 6.19(\mathrm{~d}, 1 \mathrm{H}, J=6.0 \mathrm{~Hz}), 7.41(\mathrm{~d}, 1 \mathrm{H}, J$ $=6.0 \mathrm{~Hz}) \cdot{ }^{13} \mathrm{C}$ NMR $\left(125 \mathrm{MHz}, \mathrm{CDCl}_{3}\right): \delta_{\mathrm{C}} 9.3,11.0,113.3,114.5$, 
140.5, 147.9. GC-MS: $m / z=96$. Anal. Calcd. for $\mathrm{C}_{6} \mathrm{H}_{8} \mathrm{O}: \mathrm{C}$, 74.97\%; H, 8.39\%. Found: C, 75.15\%; H, 8.26\%.

\subsection{General procedure for the synthesis of the cycloocta[b]furans(6m-6o)}

Dry triethylamine $(5 \mathrm{~mL})$ was added to a mixture of 2-bromo-cyclooct-2-enol $3 d \quad(1.0 \mathrm{mmol}), \quad \mathrm{Pd}\left(\mathrm{PPh}_{3}\right)_{2} \mathrm{Cl}_{2} \quad$ (5 mol\%) and $\mathrm{CuI}(5 \mathrm{~mol} \%)$ in an oven-dried flask under $\mathrm{N}_{2}$, and the resulting suspension was stirred for $5 \mathrm{~min}$. Terminal alkyne $4(1.2 \mathrm{mmol})$ was then added to the reaction in a dropwise manner, and the resulting mixture was stirred at room temperature until it reached completion, indicated by the disappearance of 2-bromo-cyclooct-2-enol by TLC $(\sim 6 \mathrm{~h})$. The reaction was diluted with water $(50 \mathrm{~mL})$ and extracted with ethyl acetate $(4 \times 15 \mathrm{~mL})$. The combined organic extracts were dried over anhydrous sodium sulfate and concentrated under reduced pressure to give the crude product. The crude was then purified by column chromatography over silica gel eluting with a 1:9 $(\mathrm{v} / \mathrm{v})$ mixture of EtOAc and petroleum ether to afford the pure product $(\mathbf{6 m}-\mathbf{6 0})$. Compound $\mathbf{6 m}$ was synthesized in the way using the catalyst and conditions depicted in Scheme 3.

2-Phenyl-4,5,6,7,8,9-hexahydrocycloocta[ $b]$ furan (6m). Colorless oil; IR $\left(\mathrm{CH}_{2} \mathrm{Cl}_{2}\right)$ : 3081, 3055, 2927, 2844, 1625, 1606, 1541, 1485, 1455, 1351, 895, $797 \mathrm{~cm}^{-1} .{ }^{1} \mathrm{H}$ NMR $(500 \mathrm{MHz}$, $\left.\mathrm{CDCl}_{3}\right): \delta_{\mathrm{H}} 1.49-1.55(\mathrm{~m}, 4 \mathrm{H}), 1.65-1.70(\mathrm{~m}, 2 \mathrm{H}), 1.75-1.80(\mathrm{~m}$, $2 \mathrm{H}), 2.55(\mathrm{t}, 2 \mathrm{H}, J=6.2 \mathrm{~Hz}), 2.85(\mathrm{t}, 2 \mathrm{H}, J=6.2 \mathrm{~Hz}), 6.41(\mathrm{~s}, 1 \mathrm{H})$, 7.15-7.20 (m, 1H),7.30-7.35 (m, 2H), 7.60-7.64 (m, 2H). ${ }^{13} \mathrm{C}$ NMR (125 MHz, $\left.\mathrm{CDCl}_{3}\right): \delta_{\mathrm{C}} 23.8,25.3,26.1,27.4,28.9,108.1$, $120.5,123.1,126.4,128.5,131.3,150.5,151.7$. GC-MS: $m / z=$ 226. Anal. Calcd. for $\mathrm{C}_{16} \mathrm{H}_{18} \mathrm{O}$ : C, 84.91\%; H, 8.02\%. Found: $\mathrm{C}$, 85.11\%; H, 7.98\%.

2-Pentyl-4,5,6,7,8,9-hexahydrocycloocta[b]furan (6n). Colorless oil; IR $\left(\mathrm{CH}_{2} \mathrm{Cl}_{2}\right): 2927,2860,1733,1520,1455,1345$, 1257, 1165, 1090, 1010, 930, $850 \mathrm{~cm}^{-1}$. ${ }^{1} \mathrm{H}$ NMR (500 MHz, $\left.\mathrm{CDCl}_{3}\right): \delta_{\mathrm{H}} 0.89(\mathrm{t}, 3 \mathrm{H}, J=6.4 \mathrm{~Hz}), 1.30-1.35(\mathrm{~m}, 4 \mathrm{H}), 1.44-1.68$ $(\mathrm{m}, 10 \mathrm{H}), 2.44-2.55(\mathrm{~m}, 4 \mathrm{H}), 2.70(\mathrm{~m}, 2 \mathrm{H}), 5.69(\mathrm{~s}, 1 \mathrm{H}) .{ }^{13} \mathrm{C}$ $\operatorname{NMR}\left(125 \mathrm{MHz}, \mathrm{CDCl}_{3}\right): \delta_{\mathrm{C}} 14.1,22.3,23.8,25.3,26.0,26.1,27.5$, 28.0, 28.1, 28.9, 31.5, 107.5, 118.5, 149.5, 152.9. GC-MS: $m / z=$ 220. Anal. Calcd. for $\mathrm{C}_{15} \mathrm{H}_{24} \mathrm{O}$ : C, 81.76\%; $\mathrm{H}, 10.98 \%$. Found: $\mathrm{C}$, 81.91\%; H, 10.92\%.

2-(3-(Benzyloxy)propyl)-4,5,6,7,8,9-hexahydrocycloocta[b] furan (6o). Colorless oil; IR $\left(\mathrm{CH}_{2} \mathrm{Cl}_{2}\right)$ : 2925, 2853, 1572, 1495, $1476,1450,1410,1361,1305,1280,1250,1210,1145,1100$, $1074,1025,983,960,905,881,858,800 \mathrm{~cm}^{-1} .{ }^{1} \mathrm{H}$ NMR $(500$ $\left.\mathrm{MHz}, \mathrm{CDCl}_{3}\right): \delta_{\mathrm{H}} 1.35-1.70(\mathrm{~m}, 8 \mathrm{H}), 1.85(\mathrm{q}, 2 \mathrm{H}, J=6.7 \mathrm{~Hz}), 2.39$ $(\mathrm{t}, 2 \mathrm{H}, J=6.3 \mathrm{~Hz}), 2.55-2.65(\mathrm{~m}, 6 \mathrm{H}), 3.45(\mathrm{t}, 2 \mathrm{H}, J=6.7 \mathrm{~Hz})$, $5.65(\mathrm{~s}, 1 \mathrm{H}), 7.20-7.29(\mathrm{~m}, 5 \mathrm{H}) .{ }^{13} \mathrm{C}$ NMR $\left(125 \mathrm{MHz}, \mathrm{CDCl}_{3}\right): \delta \mathrm{c}$ 23.7, 24.6, 25.3, 25.8, 26.0, 27.3, 28.2, 28.7, 69.4, 73.0, 108.0, 118.6, 127.5, 127.6, 127.7, 128.4, 128.5, 138.5, 149.7, 152.0. GC-MS: $m / z=321$. Anal. Calcd. for $\mathrm{C}_{20} \mathrm{H}_{26} \mathrm{O}_{2}$ : C, $80.50 \%$; 8.78\%. Found: C, 80.21\%; H, 8.85\%.

\subsection{Experimental procedure for the preparation of} 3-iodo-2-phenyl-4,5,6,7,8,9-hexahydrocycloocta[b]furan $8 \boldsymbol{a}$

Dry triethylamine $(50 \mathrm{~mL})$ was added to a mixture of 2-bromo-cyclooct-2-enol 3d (10.0 mmol), $\mathrm{Pd}\left(\mathrm{PPh}_{3}\right)_{2} \mathrm{Cl}_{2} \quad$ (5

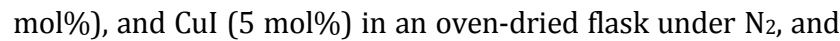
the resulting suspension was stirred for $5 \mathrm{~min}$. Phenylacetylene 4a (1.2 eq.) was then added to the reaction in a dropwise manner, and the resulting mixture was stirred at room temperature for $6 \mathrm{~h}$. Molecular iodine (1.0 eq.) was then added to the reaction, and the resulting mixture was stirred for further 2 h. The mixture was then quenched with a saturated solution of sodium thiosulfate and extracted with EtOAc $(3 \times 50 \mathrm{~mL})$. The combined organic extracts were dried over anhydrous sodium sulfate, filtered and concentrated under reduced pressure to afford the crude product, which was purified by column chromatography over silica gel (100-200 mesh) eluting cyclohexane/EtOAc to afford pure $\mathbf{8 a}$ as a colorless liquid. IR (neat): 3084, 3059, 2929, 2851, 1635, 1610, 1549, 1489, $1449 \mathrm{~cm}^{-1} .{ }^{1} \mathrm{H}$ NMR (500 MHz, $\left.\mathrm{CDCl}_{3}\right): \delta_{\mathrm{H}} 1.65-1.79(\mathrm{~m}, 4 \mathrm{H}) ; 1.81-1.92(\mathrm{~m}$, $4 \mathrm{H}) ; 2.37(\mathrm{t}, 2 \mathrm{H}, J=6.1 \mathrm{~Hz}) ; 2.72(\mathrm{t}, 2 \mathrm{H}, J=6.2 \mathrm{~Hz}) ; 7.34(\mathrm{t}, 1 \mathrm{H}, J$ $=7.6 \mathrm{~Hz}) ; 7.48(\mathrm{t}, 2 \mathrm{H}, J=7.6 \mathrm{~Hz}) ; 8.02(\mathrm{~d}, 2 \mathrm{H}, J=7.6 \mathrm{~Hz}) .{ }^{13} \mathrm{C}$ NMR $\left(125 \mathrm{MHz}, \mathrm{CDCl}_{3}\right): \delta_{\mathrm{C}} 20.7,21.9,22.9,23.4,23.6,66.9$, 123.7, 126.5, 128.0, 128.9, 129.0, 131.2, 150.1, 151.5. GC-MS: $m / z=352$. Anal. Calcd. for $\mathrm{C}_{16} \mathrm{H}_{17} \mathrm{IO}: \mathrm{C}, 54.56 \% ; \mathrm{H}, 4.86 \%$. Found: C, 53.91\%; H, 4.95\%.

\subsection{Experimental procedure for the Negishi coupling of $\mathbf{8 a}$}

A solution of organozinc reagent 7a (1.05 eq.) in THF was added to a stirred solution of $\mathbf{8 a}(1.0 \mathrm{mmol})$ and $\mathrm{Pd}\left(\mathrm{Ph}_{3} \mathrm{P}\right)_{4}(10$ mol\%) in dry dioxane at $0{ }^{\circ} \mathrm{C}$, and the resulting mixture was stirred at $25{ }^{\circ} \mathrm{C}$ for $30 \mathrm{~min}$. The reaction was quenched with 0.5 mol/L $\mathrm{HCl}$ and extracted with $\mathrm{Et}_{2} \mathrm{O}$. The combined organic extracts were then washed sequentially with saturated $\mathrm{NaHCO}_{3}$ solution and brine, and dried over anhydrous $\mathrm{Na}_{2} \mathrm{SO}_{4}$. The solvent was then removed under reduced pressure to give the crude product, which was purified by column chromatography over silica gel (100-200 mesh) eluting with a mixture of hexane and EtOAc to give the pure product $9 \mathbf{a}$ as a colorless solid. MP 72-74 ${ }^{\circ} \mathrm{C}$; IR (KBr): 3069, 3025, 2935, 1519, 1489, 1449, 1375, $781 \mathrm{~cm}^{-1} .{ }^{1} \mathrm{H}$ NMR $\left(500 \mathrm{MHz}, \mathrm{CDCl}_{3}\right): \delta_{\mathrm{H}} 1.34-1.45(\mathrm{~m}, 4 \mathrm{H})$, 1.67-1.77 (m, 4H). $2.36(\mathrm{~s}, 3 \mathrm{H}), 2.47(\mathrm{t}, 2 \mathrm{H}, J=7.6 \mathrm{~Hz}), 2.89(\mathrm{t}$, $2 \mathrm{H}, J=8.1 \mathrm{~Hz}), 6.95-7.04(\mathrm{~m}, 2 \mathrm{H}), 7.12(\mathrm{t}, 1 \mathrm{H}, J=7.6 \mathrm{~Hz})$, 7.29-7.40 (m, 4H), 7.51-7.67 (m, 2H). ${ }^{13} \mathrm{C}$ NMR (125 MHz, $\left.\mathrm{CDCl}_{3}\right): \delta \mathrm{c} 21.5,22.9,24.1,25.5,27.0,30.5,57.4,115.1,116.5$, 119.5, 120.2, 124.1, 127.9, 129.8, 130.4, 132.5, 131.9, 146.7, 150.9, 160.1. GC-MS: $m / z=316$. Anal. Calcd. for $\mathrm{C}_{23} \mathrm{H}_{24} \mathrm{O}: \mathrm{C}$, 87.30\%; H, 7.64\%. Found: C, 88.11\%; H, 7.55\%.

\subsection{Experimental procedure for the Sonogashira coupling of $\mathbf{8 a}$}

Dry triethylamine (5mL) was added to a mixture of compound $8 \mathrm{a}(1.0 \mathrm{mmol}), \mathrm{Pd}\left(\mathrm{PPh}_{3}\right)_{2} \mathrm{Cl}_{2}(5 \mathrm{~mol} \%)$ and $\mathrm{CuI}(5 \mathrm{~mol} \%)$ were placed in an oven-dried flask under $\mathrm{N}_{2}$, and the resulting suspension was stirred for $5 \mathrm{~min}$. Phenylacetylene 4 a (1.1 eq.) was added to the reaction in a dropwise manner, and the resulting mixture was stirred at room temperature for $3 \mathrm{~h}$. The reaction was diluted with water $(50 \mathrm{~mL})$ and extracted with ethyl acetate $(4 \times 15 \mathrm{~mL})$. The combined organic extracts were dried over anhydrous sodium sulfate and concentrated to dry- 
ness to give the crude product, which was purified by column chromatography over silica gel eluting with a mixture of EtOAc and petroleum ether to afford the pure product $10 \mathrm{a}$ as a yellow solid. MP 110-112 ${ }^{\circ} \mathrm{C}$; IR (KBr): 3060, 3018, 2939, 2855, 2219, $1642,1608,1556,1499,1351 \mathrm{~cm}^{-1}$. ${ }^{1} \mathrm{H}$ NMR (500 MHz, CDCl $)$ : $\delta_{\mathrm{H}} 1.66-1.79(\mathrm{~m}, 4 \mathrm{H}), 1.85-1.93(\mathrm{~m}, 4 \mathrm{H}), 2.55(\mathrm{t}, 2 \mathrm{H}, J=6.7 \mathrm{~Hz})$, $2.69(\mathrm{t}, 2 \mathrm{H}, J=6.4 \mathrm{~Hz}), 7.29(\mathrm{t}, 1 \mathrm{H}, J=7.6 \mathrm{~Hz}), 7.35-7.41(\mathrm{~m}$, $5 \mathrm{H}), 7.51-7.55(\mathrm{~m}, 2 \mathrm{H}), 8.05(\mathrm{~d}, 2 \mathrm{H}, J=8.1 \mathrm{~Hz}) .{ }^{13} \mathrm{C}$ NMR $(125$ $\left.\mathrm{MHz}, \mathrm{CDCl}_{3}\right): \delta \mathrm{c} 20.7,21.0,21.3,22.8,23.1,23.4,84.1,97.5$, $105.2,123.6,124.5,124.9,127.9,128.4,128.8,129.6,131.5$, $132.2,151.1,153.4$. GC-MS: $m / z=326$. Anal. Calcd. for $\mathrm{C}_{24} \mathrm{H}_{22} \mathrm{O}$ : C, 88.31\%; H, 6.79\%. Found: C, 88.91\%; H, 6.65\%.

\subsection{Experimental procedure for the Suzuki coupling of $\mathbf{8 a}$}

To a stirred solution of iodofuran 8 a $(1.0 \mathrm{mmol})$ in dimethoxyethane at room temperature was added phenylboronic acid 11a (1.0 eq.), $\mathrm{Pd}\left(\mathrm{PPh}_{3}\right)_{4}(10 \mathrm{~mol} \%)$ and $\mathrm{Cs}_{2} \mathrm{CO}_{3}$ (1.0 eq.), and the resulting mixture was stirred at $60^{\circ} \mathrm{C}$ for $1 \mathrm{~h}$. The mixture was cooled to room temperature and filtered through a sintered crucible. The filtrate was collected and concentrated to dryness under reduced pressure to give the crude product, which was purified by column chromatography over silica gel eluting with a mixture of EtOAc and petroleum ether to afford the pure product $12 \mathrm{a}$ as a colorless solid. MP $88-90{ }^{\circ} \mathrm{C}$; IR (KBr): 3068, 3025, 2932, 1515, 1475, 1381, $763 \mathrm{~cm}^{-1}$. ${ }^{1} \mathrm{H} \mathrm{NMR}$ $\left(500 \mathrm{MHz}, \mathrm{CDCl}_{3}\right): \delta_{\mathrm{H}} 1.65-1.78(\mathrm{~m}, 4 \mathrm{H}), 1.83-1.92(\mathrm{~m}, 4 \mathrm{H})$, $2.39(\mathrm{t}, 2 \mathrm{H}, J=6.7 \mathrm{~Hz}), 2.75(\mathrm{t}, 2 \mathrm{H}, J=6.6 \mathrm{~Hz}), 6.78-6.89(\mathrm{~m}, 2 \mathrm{H})$, 7.17-7.35 (m, 5H), 7.44-7.55 (m, 3H). ${ }^{13} \mathrm{C}$ NMR $(125 \mathrm{MHz}$, $\left.\mathrm{CDCl}_{3}\right): \delta_{\mathrm{c}} 20.9,21.3,22.7,22.9,23.1,23.4,55.7,114.1,114.2$, 119.8, 122.1, 125.6, 126.5, 126.6, 127.4, 128.5, 130.8, 131.9, 146.8, 150.5, 158.9. GC-MS: $m / z=302$. Anal. Calcd. for $\mathrm{C}_{22} \mathrm{H}_{22} \mathrm{O}$ : C, $87.38 \%$; H, 7.33\%. Found: C, 86.97\%; H, 7.45\%.

\subsection{Experimental procedure for the Stille coupling of $\mathbf{8 a}$}

To a stirred solution of iodofuran $\mathbf{8 a}(1.0 \mathrm{mmol})$ in dry THF $(10 \mathrm{~mL})$ at room temperature was added $\mathrm{Pd}\left(\mathrm{PPh}_{3}\right)_{4}(10 \mathrm{~mol} \%)$, $\mathrm{LiCl}$ (3.0 eq.) and tributyl(vinyl)tin (2.0 eq.), and the resulting mixture was stirred at room temperature for $12 \mathrm{~h}$. The mixture was filtered through a pad of Celite and the filtrate was concentration under reduced pressure to give a residue, which was purified by column chromatography over silica gel (100-200 mesh) eluting with a mixture of hexane and EtOAc to afford 14a as a colorless liquid. IR (neat): 3091, 3061, 3035, 2935, 2849, 1631, 1614, 1591, 1471, 1455, 1425, 1345, $781 \mathrm{~cm}^{-1} .{ }^{1} \mathrm{H}$ NMR (500 MHz, $\left.\mathrm{CDCl}_{3}\right): \delta_{\mathrm{H}} 1.65-1.79(\mathrm{~m}, 4 \mathrm{H}), 1.85-1.94(\mathrm{~m}$, $4 \mathrm{H}), 2.55-2.67(\mathrm{~m}, 4 \mathrm{H}), 5.35\left(\mathrm{dd}, 1 \mathrm{H}, J_{1}=12.2 \mathrm{~Hz} ; J_{2}=2.1 \mathrm{~Hz}\right)$, $5.47\left(\mathrm{dd}, 1 \mathrm{H}, J_{1}=18.4 \mathrm{~Hz} ; J_{2}=12.2 \mathrm{~Hz}\right.$ ), 6.91 (dd, $1 \mathrm{H}, J_{1}=18.2$ $\left.\mathrm{Hz}, J_{2}=12.2 \mathrm{~Hz}\right), 7.29-7.34(\mathrm{~m}, 1 \mathrm{H}), 7.40-7.45(\mathrm{~m}, 2 \mathrm{H}), 7.67(\mathrm{t}$, $2 \mathrm{H}, J=7.6 \mathrm{~Hz}) .{ }^{13} \mathrm{C}$ NMR $\left(125 \mathrm{MHz} \mathrm{CDCl}_{3}\right): \delta \mathrm{c} 21.5,22.6,22.9$, 23.4, 115.2, 118.3, 127.1, 128.8, 129.6, 130.1, 130.3, 131.9, 149.5, 150.6. GC-MS: $m / z=252$. Anal. Calcd. for $\mathrm{C}_{18} \mathrm{H}_{20} \mathrm{O}: \mathrm{C}$, 85.67\%; H, 7.99\%. Found: C, 86.07\%; H, 7.87\%.

\subsection{Experimental procedure for the Heck coupling of $\mathbf{8 a}$}

To a stirred solution of iodofuran 8 a (1.0 mmol) in DMAc $(1.5 \mathrm{~mL})$ at room temperature was added ethylacrylate $(1.1$ eq.), $\mathrm{Pd}(\mathrm{OAc})_{2}(5 \mathrm{~mol} \%), n \mathrm{Bu} 3 \mathrm{P}(25 \mathrm{~mol} \%)$ and pyridine $(3.0$ eq.), and the resulting mixture was stirred at $50{ }^{\circ} \mathrm{C}$ for $1 \mathrm{~h}$. The mixture was then diluted with brine $(10 \mathrm{~mL})$ and extracted with EtOAc $(3 \times 15 \mathrm{~mL})$. The combined organic extracts were washed with brine $(20 \mathrm{~mL})$ and dried over anhydrous $\mathrm{MgSO}_{4}$. The solvent was then removed under reduced pressure to give a residue, which was purified by column chromatography over silica gel eluting with a mixture of hexane and EtOAc to give 16a as a pale yellow liquid. IR (neat): 3095, 3061, 2941, 2841, $1717,1635,1621,1542,1480,1455,1425,1341,781 \mathrm{~cm}^{-1} .{ }^{1} \mathrm{H}$ NMR (500 MHz, CDCl $): \delta_{\mathrm{H}} 1.29(\mathrm{t}, 3 \mathrm{H}, J=7.6 \mathrm{~Hz}), 1.66-1.78(\mathrm{~m}$, $4 \mathrm{H}), 1.81-1.92(\mathrm{~m}, 4 \mathrm{H}), 2.67-2.75(\mathrm{~m}, 4 \mathrm{H}), 3.96(\mathrm{q}, 2 \mathrm{H}, J=7.6$ $\mathrm{Hz}), 6.52(\mathrm{~d}, 1 \mathrm{H}, J=16.4 \mathrm{~Hz}), 7.30(\mathrm{t}, 1 \mathrm{H}, J=7.6 \mathrm{~Hz}), 7.45(\mathrm{t}, 2 \mathrm{H}, J$ $=7.6 \mathrm{~Hz}), 7.65(\mathrm{t}, 2 \mathrm{H}, J=7.6 \mathrm{~Hz}), 7.95(\mathrm{~d}, 1 \mathrm{H}, J=16.4 \mathrm{~Hz}) .{ }^{13} \mathrm{C}$ NMR (125 MHz, $\mathrm{CDCl}_{3}$ ): $\delta_{\mathrm{C}} 13.8,21.8,22.4,22.7,23.0,23.3,51.5$, 61.7, 117.1, 117.5, 117.9, 127.2, 128.0, 128.6, 130.8, 137.5, 151.8, 153.8, 167.1. GC-MS: $m / z=324$. Anal. Calcd. for $\mathrm{C}_{21} \mathrm{H}_{24} \mathrm{O}_{3}$ : C, 77.75\%; H, 7.46\%. Found: $\mathrm{C}, 77.57 \%$; $\mathrm{H}, 7.53 \%$.

\subsection{Experimental for the one-pot synthesis of compound $\mathbf{1 8 a}$}

$\mathrm{AuBr}_{3}$ (5 mol\%) was added to a solution of compound $\mathbf{6 f}$ $(1.0 \mathrm{mmol})$ in dichloroethane $(1 \mathrm{~mL})$ under $\mathrm{N}_{2}$ at room temperature, and the resulting mixture was heated at $70{ }^{\circ} \mathrm{C}$ for $30 \mathrm{~min}$. Butenone 17a (1.2 eq.) was then added to the reaction, and the resulting mixture was stirred for $20 \mathrm{~min}$. Upon completion of the reaction, as indicated by TLC, the reaction mixture was quenched with water $(10 \mathrm{~mL})$ and extracted with dichloromethane $(3 \times 5 \mathrm{~mL})$. The combined organic extracts were dried over anhydrous $\mathrm{Na}_{2} \mathrm{SO}_{4}$, filtered and concentrated under reduced pressure to afford the crude product, which was purified by column chromatography over silica gel (100-200 mesh) eluting with a mixture of cyclohexane and EtOAc to afford pure product 18a as a yellow liquid. IR (neat): 3098, 3055, 2946, $1685,1586,1355 \mathrm{~cm}^{-1}$. ${ }^{1} \mathrm{H}$ NMR $\left(500 \mathrm{MHz}, \mathrm{CDCl}_{3}\right): \delta_{\mathrm{H}} 1.78-1.85$ (m, 2H), 1.88-1.95 (m, 2H), $2.19(\mathrm{~s}, 3 \mathrm{H}), 2.45(\mathrm{t}, 2 \mathrm{H}, J=6.9 \mathrm{~Hz})$, $2.61(\mathrm{t}, 2 \mathrm{H}, J=6.7 \mathrm{~Hz}), 2.81(\mathrm{t}, 2 \mathrm{H}, J=3.4 \mathrm{~Hz}), 2.95(\mathrm{t}, 2 \mathrm{H}, J=2.8$ $\mathrm{Hz}), 7.29(\mathrm{t}, 1 \mathrm{H}, J=7.6 \mathrm{~Hz}), 7.45(\mathrm{t}, 2 \mathrm{H}, J=7.6 \mathrm{~Hz}), 7.66-7.77(\mathrm{~m}$, 2H). ${ }^{13} \mathrm{C}$ NMR $\left(125 \mathrm{MHz}, \mathrm{CDCl}_{3}\right): \delta_{\mathrm{C}} 19.5,20.5,21.4,22.9,23.6$, 30.1, 44.2, 118.2, 119.1, 120.1, 121.1, 123.5, 124.3, 128.5, 150.3, 151.4, 208.8. GC-MS: $m / z=268$. Anal. Calcd. for $\mathrm{C}_{18} \mathrm{H}_{20} \mathrm{O}_{2}: \mathrm{C}$, 80.56\%; H, 7.51\%. Found: C, 81.07\%; H, 7.39\%.

\subsection{Experimental procedure for the one-pot synthesis of compound $19 a$}

To a mixture of $\mathrm{AuBr}_{3}$ (5 mol\%) and $\mathrm{AgSbF}_{6}$ (5 mol\%) in dry dichloroethane $(1 \mathrm{~mL})$ under $\mathrm{N}_{2}$ was added a solution of compound $\mathbf{6 f}(1.0 \mathrm{mmol})$ in dichloroethane $(1 \mathrm{~mL})$, and the resulting mixture was heated at $70{ }^{\circ} \mathrm{C}$ for $30 \mathrm{~min}$. Phenylacetylene $\mathbf{4 a}$ (1.2 eq.) was added to the reaction, and the resulting mixture was stirred for $3 \mathrm{~h}$. The reaction was then cooled to room temperature and quenched with water $(10 \mathrm{~mL})$. The resulting mixture was extracted with dichloromethane $(3 \times 5 \mathrm{~mL})$ and the combined organic layers were dried over anhydrous 
$\mathrm{Na}_{2} \mathrm{SO}_{4}$. The solvent was then removed under reduced pressure to afford the crude product, which was purified by column chromatography over silica gel (100-200 mesh) eluting with a mixture of cyclohexane and EtOAc to afford pure product 19a as colorless liquid. IR (neat): 3095, 3054, 2941, 1685, 1609, 1450, 1355, 898, $747 \mathrm{~cm}^{-1} .{ }^{1} \mathrm{H}$ NMR $\left(500 \mathrm{MHz}^{\mathrm{CDCl}} 3\right): \delta_{\mathrm{H}}$ $1.77-1.85(\mathrm{~m}, 2 \mathrm{H}), 1.89-1.96(\mathrm{~m}, 2 \mathrm{H}), 2.65(\mathrm{t}, 2 \mathrm{H}, J=6.4 \mathrm{~Hz})$, $2.78(\mathrm{t}, 2 \mathrm{H}, J=6.6 \mathrm{~Hz}), 5.41(\mathrm{~d}, 1 \mathrm{H}, J=2.3 \mathrm{~Hz}), 5.67(\mathrm{~d}, 1 \mathrm{H}, J=$ $2.0 \mathrm{~Hz}), 7.15-7.29(\mathrm{~m}, 4 \mathrm{H}), 7.35-7.48(\mathrm{~m}, 5 \mathrm{H}), 7.49-7.54(\mathrm{~m}$, 1H). ${ }^{13} \mathrm{C}$ NMR $\left(125 \mathrm{MHz}, \mathrm{CDCl}_{3}\right): \delta_{\mathrm{C}} 19.5,22.5,23.4,23.9,105.5$, $120.1,121.2,124.4,127.5,127.9,128.1,128.3,128.6,129.3$, 141.2, 145.8, 150.4, 150.6. GC-MS: $m / z=300$. Anal. Calcd. for $\mathrm{C}_{22} \mathrm{H}_{20} \mathrm{O}$ : C, 87.96\%; H, 6.71\%. Found: C, 88.17\%; H, 6.63\%.

\section{Results and discussion}

We previously reported a convenient method for the synthesis of fused furans via $\mathrm{AuBr}_{3}$-catalyzed cycloisomerization of 2-alkynyl-cycloalk-2-enols (Scheme 1) [24]. The requisite 2-alkynyl-cycloalk-2-enols were synthesized via the $\alpha$-iodination of the corresponding cycloalken-2-ones $\mathbf{1}$ with I2/pyridine, which afforded the 2-iodocycloalk-2-enones $\mathbf{2}$ [64-66]. Subsequent 1,2-reduction of these 2-iodocycloalk-2enones 2 with a stoichiometric mixture of $\mathrm{NaBH}_{4}$ and $\mathrm{CeCl}_{3} \cdot 7 \mathrm{H}_{2} \mathrm{O}$ gave the 2 -iodoenols $3[67,68]$. These iodoenols were then treated with a variety of terminal alkynes $\mathbf{4}$ under Sonogashira conditions $[69,70]$ to give the hitherto unknown 2-alkynyl-cyclo-alk-2-enols $\mathbf{5}$ in excellent yields. A wide range of coupling products were successfully synthesized under these conditions, including those bearing aliphatic, aromatic and heteroaromatic substituents, with yields ranging from good to excellent (Table 1).

The results of a series of optimization studies revealed that all of the substrates underwent the desired cycloisomerization reaction in the presence of $5 \mathrm{~mol} \% \mathrm{AuBr}_{3}$ in 1,2-dichloroethane (Table 2). As shown in Table 2, the yields of the fused furans varied from good to excellent depending on the nature of the substituent attached to the alkyne moiety and the reaction temperature. Furthermore, the results indicated that the efficiency of the cyclization was dependent on the nature and the size of the ring attached to the alkyne moiety for substrates 5a-5l. For example, substrates bearing a cycloheptyl ring, the
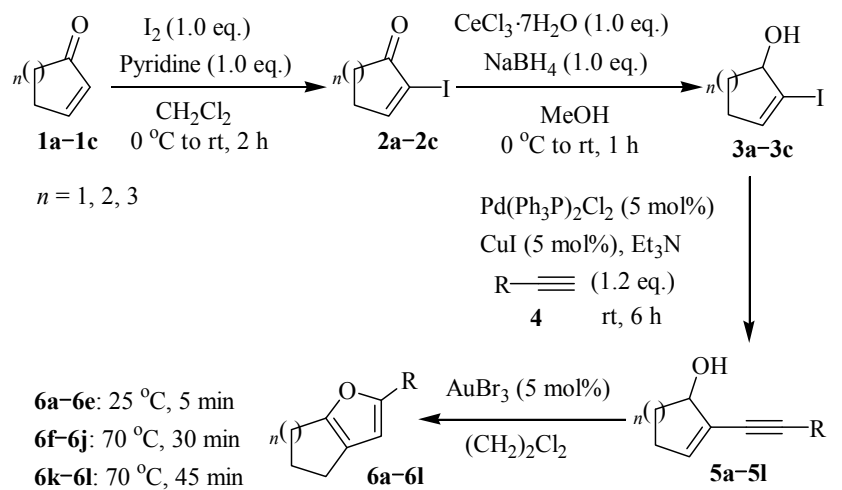

Scheme 1. AuBr 3 -catalyzed synthesis of furans 6a-6l.
Table 1

Synthesis of acetylenic alcohols.

\begin{tabular}{|c|c|c|c|c|}
\hline Entry & $n$ & R 4 & $5^{\text {a }}$ & Yield ${ }^{\mathrm{b}}(\%)$ \\
\hline 1 & 3 & phenylacetylene (4a) & $5 \mathbf{a}$ & 90 \\
\hline 2 & 3 & 1-ethynyl-4-pentylbenzene (4b) & $\mathbf{5 b}$ & 92 \\
\hline 3 & 3 & 2-ethynylthiopene (4c) & $5 c$ & 88 \\
\hline 4 & 3 & 1-hexyne (4d) & $\mathbf{5 d}$ & 89 \\
\hline 5 & 3 & 1-ethynylcyclohexanol (4e) & $5 e$ & 77 \\
\hline 6 & 2 & phenylacetylene (4a) & $5 f$ & 91 \\
\hline 7 & 2 & 1-ethynyl-4-pentylbenzene (4b) & $5 g$ & 94 \\
\hline 8 & 2 & 2-ethynylpyridine (4f) & $5 h$ & 79 \\
\hline 9 & 2 & 1-hexyne (4g) & $5 \mathbf{i}$ & 89 \\
\hline 10 & 2 & 1-ethynylcyclohexanol (4e) & $5 \mathbf{j}$ & 79 \\
\hline 11 & 1 & phenylacetylene (4a) & $5 \mathbf{k}$ & 80 \\
\hline 12 & 1 & 1-ethynyl-4-pentylbenzene (4b) & 51 & 81 \\
\hline
\end{tabular}

reaction proceeds smoothly over a short reaction time, even at room temperature (Table 2, entries 1-5). Substrates bearing a cyclohexyl ring also reacted rapidly to produce the desired products in good yields when the reaction was carried out for $30 \mathrm{~min}$ at $70{ }^{\circ} \mathrm{C}$ (Table 2, entries 6-10). However, substrates with a cyclopentyl ring reacted slowly to give low yields of the desired products, even when the reaction time was extended to 45 min (Table 2, entries 11 and 12). These observations can be rationalized in terms of the greater degree of strain associated with the resulting cyclopenta[$[b]$ furan products compared with the cyclohexa $[b]$ furan and cyclohepta $[b]$ furan products. The results of these experiments also revealed that the nature of the substituent on the alkyne of the 2-alkynylcycloalk-2-enol substrate did not play a significant role in determining the success of the cyclization, because aliphatic, aromatic and heteroaromatic groups were well tolerated at this position to give almost identical yields of the products. The formation of the products was confirmed by the appearance of a singlet peak in their ${ }^{1} \mathrm{H}$ NMR spectra in $\mathrm{CDCl}_{3}$ with a $\delta_{\mathrm{H}}$ value in the range of 6.20-6.30 ppm, which was attributed to the $\mathrm{C} 3-\mathrm{H}$ of the furan ring. Moreover, the ${ }^{13} \mathrm{C}$ NMR spectra of these products all contained a peak with a $\delta$ c value in the range of 104-109 ppm, which con-

Table 2

Synthesis of furans 6a-6l.

\begin{tabular}{lccc}
\hline Entry & $\mathbf{5}$ & $\mathbf{6}^{\mathrm{a}}$ & Yield $^{\mathrm{b}}(\%)$ \\
\hline 1 & $\mathbf{5 a}$ & $\mathbf{6 a}$ & 90 \\
2 & $\mathbf{5 b}$ & $\mathbf{6 b}$ & 91 \\
3 & $\mathbf{5 c}$ & $\mathbf{6 c}$ & 89 \\
4 & $\mathbf{5 d}$ & $\mathbf{6 d}$ & 95 \\
5 & $\mathbf{5 e}$ & $\mathbf{6 e}$ & 79 \\
6 & $\mathbf{5 f}$ & $\mathbf{6 f}$ & 86 \\
7 & $\mathbf{5 g}$ & $\mathbf{6 g}$ & 88 \\
8 & $\mathbf{5 h}$ & $\mathbf{6 h}$ & 70 \\
9 & $\mathbf{5 i}$ & $\mathbf{6 i}$ & 92 \\
10 & $\mathbf{5 j}$ & $\mathbf{6 j}$ & 83 \\
11 & $\mathbf{5 k}$ & $\mathbf{6 k}$ & 60 \\
12 & $\mathbf{5 l}$ & $\mathbf{6 l}$ & 66 \\
\hline a All of these products were characterized by IR, ${ }^{1} \mathrm{H}$ NMR, ${ }^{13} \mathrm{C}$ NMR and \\
GC-MS analyses \\
b Isolated yield after column chromatography.
\end{tabular}


firmed the presence of the C3 carbon of the furan ring.

In light of their biological significance and their prevalence as the core substructures in numerous crenulide diterpenoid natural products, we became interested in extending the scope of our study to cyclooctyl-fused furan ring systems [71,72]. Using the optimized reaction conditions, we initially explored the general scope of our methodology by investigating the synthesis of a series of cyclooctyl furans (Scheme 2). According to the literature, the precursors for this cyclization reaction could be synthesized from commercially available cycloheptene (1d) $[73,74]$. Thus, the cyclopropanation of $\mathbf{1 d}$ with $\mathrm{CHBr}_{3}$ afforded cycloadduct 2d, which underwent a ring expansion reaction with $\mathrm{AgClO}_{4}$ in aqueous acetone to furnish the 2-bromoenol 3d [75]. Surprisingly, the subsequent alkynylation of $\mathbf{3 d}$ under the standard Sonogashira conditions did not lead to the expected alkynylated product $\mathbf{5}$. In contrast to other ring systems $(n=1$, $2,3)$, we found that the eight-membered ring $\mathbf{3 d}$ reacted rapidly under the Sonogashira condition to afford the cyclized product 6 directly without the isolation of the cross-coupled intermediate $\mathbf{5}$.

At this stage in the study, the identity of the catalytic species involved in this one-pot operation was unknown. As well as playing an important role in oxidative addition reactions $[76,77]$, palladium salts can catalyze the intramolecular cyclization reactions of alkynes bearing a hydroxyl tether [78-81]. Prompted by this literature survey, we assumed that palladium was the catalyst involved in this tandem process. To test this hypothesis, we investigated the synthesis and isolation of alkyne $\mathbf{5 m}$, which was readily prepared by the Castro-Stephens coupling of 2-bromoenol 3d with phenylethynylcopper(I) [82] (Scheme 3). This reaction led to the stable alkyne $\mathbf{5 m}$ in excellent yield without the formation of any of the corresponding cyclized product $6 \mathbf{m}$, which suggested that $\mathrm{Et}_{3} \mathrm{~N}$ and $\mathrm{CuBr}$ (formed as by-product) were not acting as catalysts for the cyclization. We subsequently investigated the cyclization of $\mathbf{5 m}$ using $5 \mathrm{~mol} \% \mathrm{PdCl}_{2}\left(\mathrm{Ph}_{3} \mathrm{P}\right)_{2}$ and found that the reaction proceeded smoothly to afford the cyclization product $\mathbf{6 m}$ in excellent yield. $\mathrm{PdCl}_{2}$ also gave the cyclized product $6 \mathbf{m}$ in excellent yield, which indicated that this reaction did not require a neutral phosphine ligand. These two test reactions clearly indicated that Pd(II) was the active catalytic species involved in this transformation. Notably, the reaction of $\mathbf{5 m}$ with CuI (co-catalyst in the Sonogashira reaction) under the same conditions led to only trace quantities of the cyclization product $\mathbf{6 m}$ with $80 \%$ of starting material being recovered unchanged. This result therefore precluded the participation of $\mathrm{CuI}$ in the cyclization reaction. Finally, the reaction of $\mathbf{5 m}$ with a catalytic amount of $\mathrm{AuBr}_{3}$ also afforded a clean conversion to product 6m, which suggested that our gold-based methodology was indeed applicable to cyclooctyl systems.

Based on these results, we proposed a mechanism for the formation of fused furans, which is shown in Scheme 4. Thus, the initial coordination of the soft Lewis acidic metal [M] to alkyne 5 would lead to $\pi$-complex 5I. The increased electrophilicity of the alkyne would allow for the nucleophilic attack of the adjoining hydroxyl group to give the cyclized intermediate 5II. The subsequent proto-deauration of 5II would results in the formation of 5III, which would undergo an isomerization
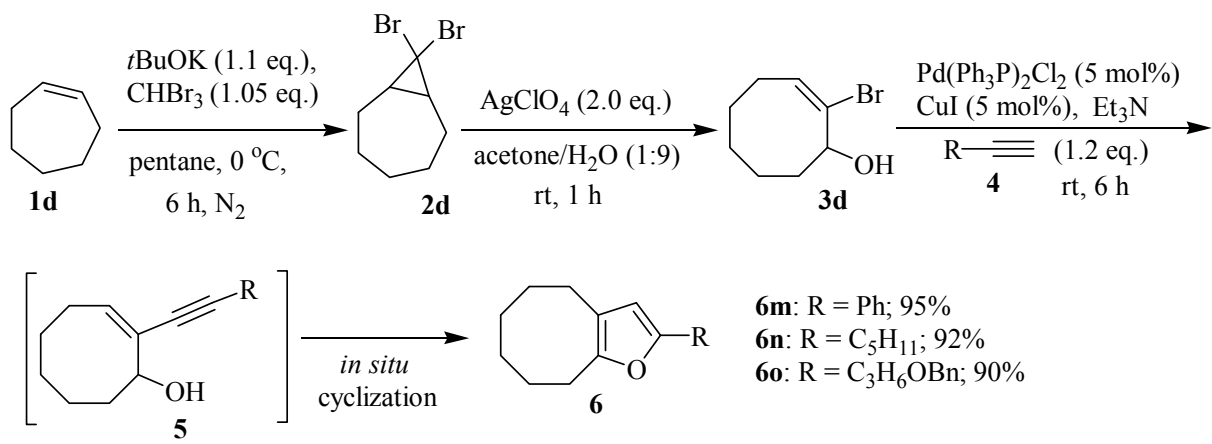

Scheme 2. Synthesis of cyclooctylfurans 6m-6o.

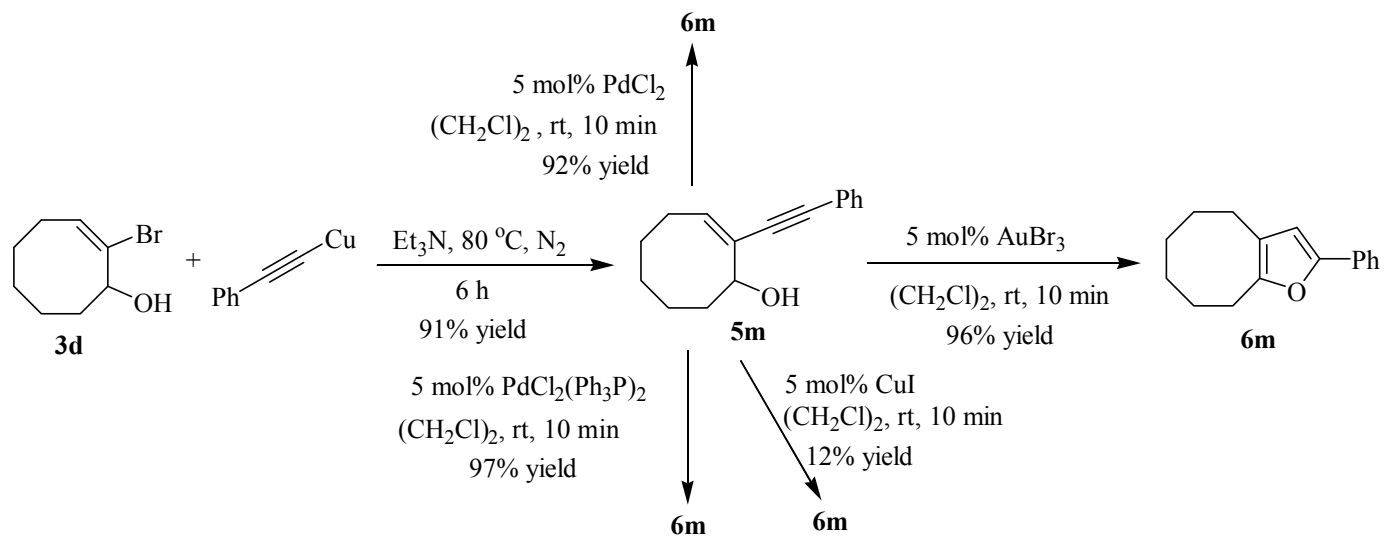

Scheme 3. Cyclization of $\mathbf{5 m}$ by several transition metal catalysts. 


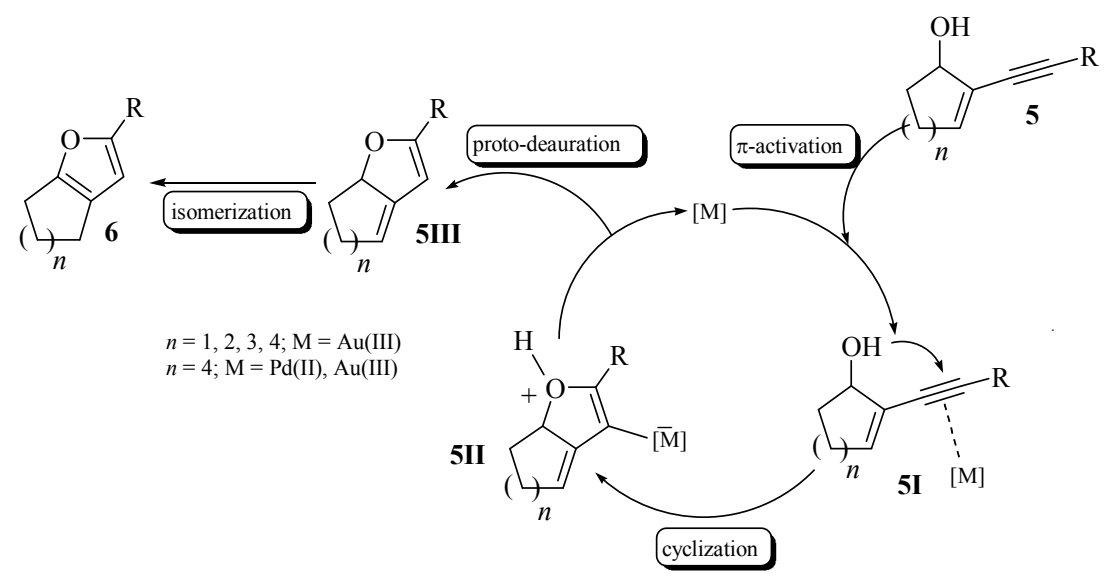

Scheme 4. Tentative mechanism for the formation of furans 6.

reaction to produce the fused furan 6 .

At this stage in the study, we decided to delineate the scope of our chemistry through a series of synthetic elaborations. Keeping in mind the versatility of the iodide functionality in organometallic transformations, we attempted the one-pot synthesis of 3-iodofuran 8a (Scheme 5). Pleasingly, this sequential process provided the desired iodide $\mathbf{8 a}$ in $84 \%$ yield. The subsequent Negishi coupling reaction of compound $\mathbf{8 a}$ with the arylzinc reagent $\mathbf{7 a}$ in the presence of $10 \mathrm{~mol} \%$ $\mathrm{Pd}\left(\mathrm{Ph}_{3} \mathrm{P}\right)_{4}$ allowed for the introduction of an aryl group, with the tetra-substituted furan product 9a being isolated in $79 \%$ yield $[83,84]$. Furthermore, the Sonogashira reaction of $\mathbf{8 a}$ with phenylacetylene (4a) provided the functionalized alkyne 10a in $88 \%$ yield [69]. The Suzuki-Miyaura coupling reaction of $\mathbf{8 a}$ with phenylboronic acid 11a afforded the 2,3-diphenyl substituted furan 12a in 90\% yield [85]. Lastly, compound 8a also underwent Stille and Heck coupling reactions with tributyl(vinyl)tin (13a) and ethylacrylate (15a), respectively, to give the corresponding 3-vinyl derivatives 14a and 16a in moderate to good yields [86,87].

Having demonstrated the catalytic application of this newly developed palladium-catalyzed tandem process, we turned our attention back towards the possibility of using $\mathrm{AuBr}_{3}$ to facilitate other interesting one-pot procedures with particular emphasis on Michael- [88] and Friedel-Crafts-type reactions [89] (Scheme 6). For example, the cyclization of substrate $\mathbf{5 f}$ under the optimized conditions followed by the in situ addition of methyl vinyl ketone 17a resulted in the formation of the Michael adduct 18a in $80 \%$ yield. Furthermore, the tandem Friedel-Crafts alkenylation of $\mathbf{5 f}$ with phenylacetylene (4a) in the presence of a silver co-catalyst resulted in product 19a bearing a $\mathrm{C}=\mathrm{C}$ bond, albeit in a low yield of $22 \%$. It is noteworthy, however, that this reaction proceeded regioselectively, with the addition only occurring at the internal

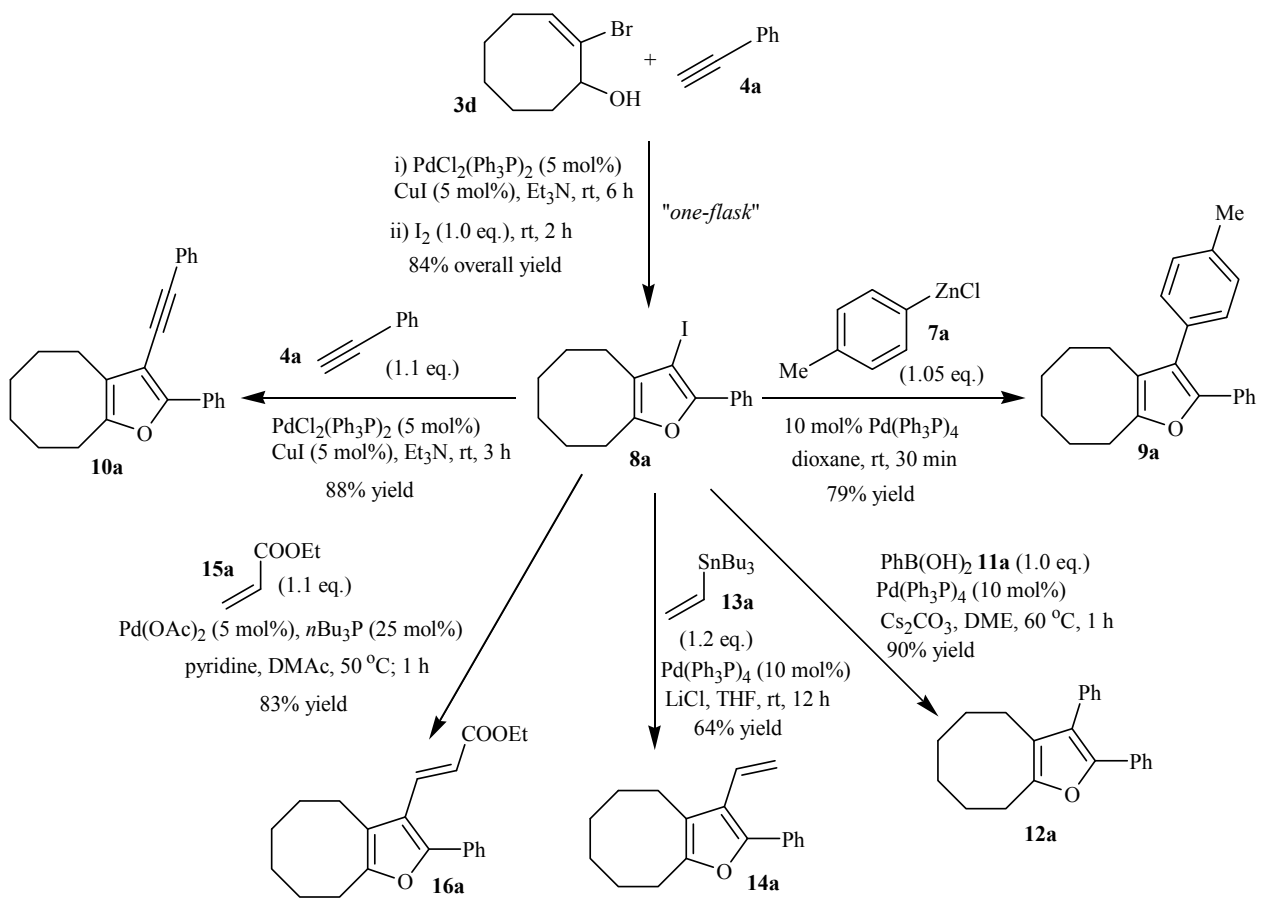

Scheme 5. Pd-catalyzed organometallic transformations of $\mathbf{8 a}$. 


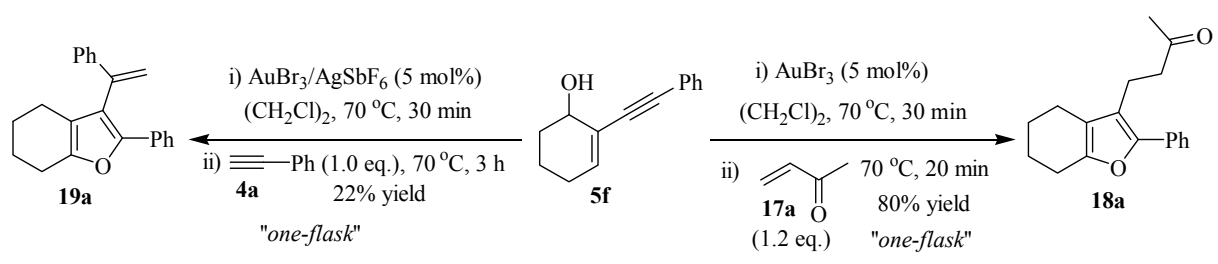

Scheme 6. $\mathrm{AuBr}_{3}$-catalyzed sequential processes.

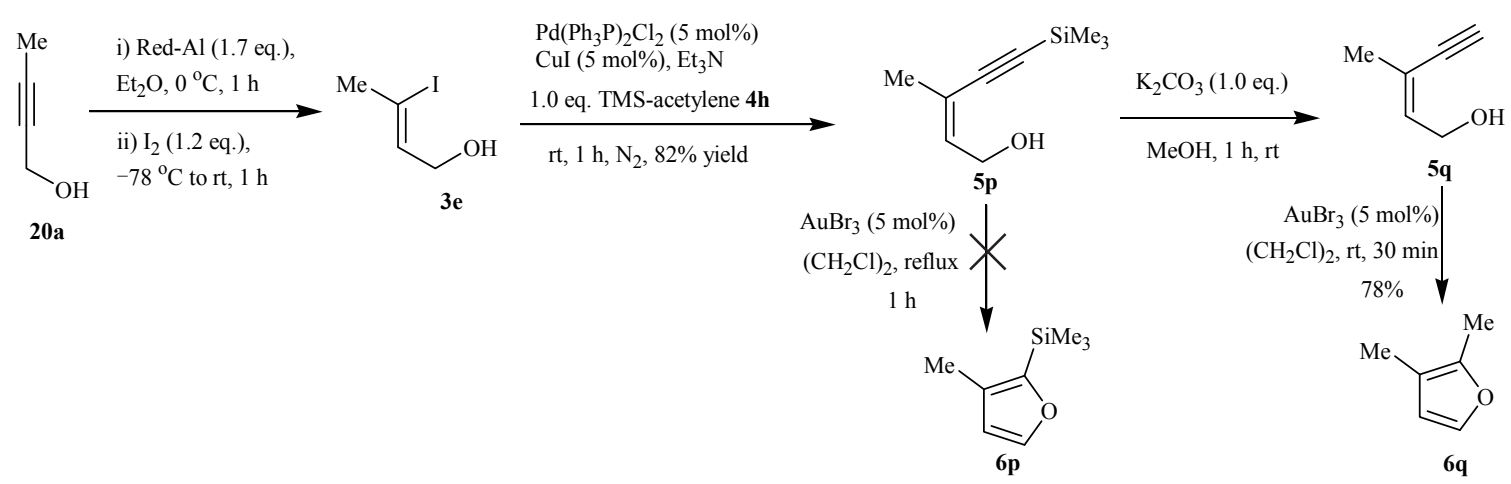

Scheme 7. $\mathrm{AuBr}_{3}$ catalyzed cyclization of acyclic substrates.

carbon of alkyne $\mathbf{4 a}$, as indicated by NMR analysis of the crude mixture. This observation was consistent with those of previous studies, in that the Au $\pi$-acid drove the addition of an arene/heteroarene to the internal carbon of the terminal acetylene [89].

Finally, we were intrigued by the prospect of applying this methodology to acyclic acetylenic alcohols for the synthesis of non-fused furan rings (see Scheme 7). The requisite (Z)-iodoallylic alcohol $\mathbf{3 e}$ was prepared by the reaction of commercially available butyn-1-ol (20a) with Red-Al $\left[\mathrm{NaAlH}_{2}\left(\mathrm{OCH}_{2} \mathrm{CH}_{2} \mathrm{OMe}\right)_{2}\right]$, followed by quenching of the resulting carbanion with $\mathrm{I}_{2}$ [90]. Subsequent Sonogashira coupling of $\mathbf{3 e}$ with ethynyltrimethylsilane $\mathbf{4 h}$ gave alkyne $\mathbf{5 p}$ [91]. Disappointingly, the reaction of silylated alkyne 5p under our validated conditions did not provide the expected product $\mathbf{6 p}$, even under reflux conditions. This result was attributed to the reduced aurophilicity of alkyne $\mathbf{5 p}$ as a result of the deactivating effect of the silyl group. However, substrate $\mathbf{5 q}$ bearing an unsubstituted terminal alkyne underwent a 5-exo-dig cyclization exclusively to give furan $\mathbf{6 q}$ as the only product. The regioselectivity of this reaction was attributed to the greater electrophilicity of the remote sp-hybridized carbon of the alkyne.

\section{Conclusions}

The use of $\mathrm{AuBr}_{3}$ as a catalyst allowed for the smooth and effective cycloisomerization of 2-alkynylcycloalk-2-enols to give the corresponding cycloalkyl-fused furans. A detailed study of this methodology as a synthetic strategy for the formation of a diverse range of furan derivatives has also been provided in terms of its scope and limitations towards ring-free substrates. We also observed the in situ cross-coupling/cycloisomerization of $\alpha$-bromocyclooct-2-enols with terminal alkynes under Sonogashira conditions and showed that Pd(II) was the active catalytic species involved in this process. This methodology was also applied to a series of tandem processes, which provided access to a wide range of functionalized furan molecules. Given that most of these substrates are commercially available or synthetically accessible this new method could be used for the synthesis of furan-based libraries for detailed SAR studies in medicinal chemistry. Further studies towards identifying new applications for this process to generate furan-based chemical libraries of potential pharmacological interest are currently underway.

\section{Acknowledgments}

C. Praveen acknowledges the Department of Science and Technology for providing INSPIRE faculty award. C. Praveen also thanks Dr. Vijayamohanan K. Pillai and Dr. D. Jeyakumar of CSIR-CECRI for providing infrastructure facilities.

\section{References}

[1] C. H. M. Amijs, C. Ferrer, A. M. Echaverren, Chem. Commun., 2007, 698.

[2] A. Hoffmann-Roder, N. Krause, Org. Biomol. Chem., 2005, 3, 387.

[3] A. S. K. Hashmi, Angew. Chem. Int. Ed., 2005, 44, 6990.

[4] A. Arcadi, S. Di Giuseppe, Curr. Org. Chem., 2004, 8, 795.

[5] R. A. Widenhoefer, X. Q. Han, Eur. J. Org. Chem., 2006, 4555.

[6] H. C. Shen, Tetrahedron, 2008, 64, 7847.

[7] Z. G. Li, C. Brouwer, C. He, Chem. Rev., 2008, 108, 3289.

[8] L. M. Zhang, J. W. Sun, S. A. Kozmin, Adv. Synth. Catal., 2006, 348, 2271.

[9] N. Asao, Synlett, 2006, 1645.

[10] S. M. Ma, S. C. Yu, Z. H. Gu, Angew. Chem. Int. Ed., 2006, 45, 200.

[11] A. M. Echavarren, C. Nevado, Chem. Soc. Rev., 2004, 33, 431.

[12] G. Dyker, Angew. Chem. Int. Ed., 2000, 39, 4237.

[13] A. S. K. Hashmi, Gold Bull., 2004, 37, 51.

[14] R. Skouta, C. J. Li, Tetrahedron, 2008, 64, 4917. 


\section{Graphical Abstract}

Chin. J. Catal., 2016, 37: 288-299 doi: 10.1016/S1872-2067(15)60994-9

Extrapolation of the gold-catalyzed cycloisomerization to the palladium-catalyzed cross-coupling/cycloisomerization of acetylenic alcohols for the synthesis of polysubstituted furans: Scope and application to tandem processes

Chandrasekar Praveen*, Paramasivan T. Perumal

Central Electrochemical Research Institute (CSIR laboratory), India; Central Leather Research Institute (CSIR laboratory), India

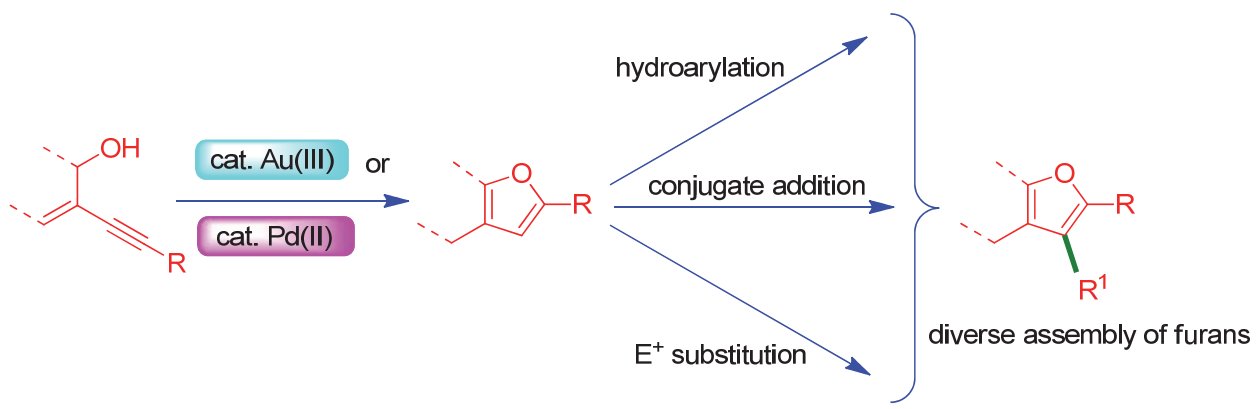

Furan derivatives have been efficiently synthesized from acetylenic alcohols using a combination of gold and palladium catalysis. The utility of this protocol has been highlighted by performing several tandem and one-pot reactions.

[15] N. D. Shapiro, F. D. Toste, Synlett, 2010, 675.

[16] A. S. K. Hashmi, G. J. Hutchings, Angew. Chem. Int. Ed., 2006, 45, 7896.

[17] D. J. Gorin, F. D. Toste, Nature, 2007, 446, 395.

[18] A. S. K. Hashmi, Chem. Rev., 2007, 107, 3180.

[19] A. Fürstner, P. W. Davies, Angew. Chem. Int. Ed., 2007, 46, 3410.

[20] M. Rudolph, A. S. K. Hashmi, Chem. Soc. Rev., 2012, 41, 2448.

[21] A. S. K. Hashmi, M. Rudolph, Chem. Soc. Rev., 2008, 37, 1766.

[22] E. Jiménez-Núñez, A. M. Echavarren, Chem. Commun., 2007, 333.

[23] A. S. K. Hashmi, Gold Bull., 2003, 36, 1.

[24] C. Praveen, P. Kiruthiga, P. T. Perumal, Synlett, 2009, 1990.

[25] C. Praveen, S. Jegatheesan, P. T. Perumal, Synlett, 2009, 2795.

[26] C. Praveen, A. Kalyanasundaram, P. T. Perumal, Synlett, 2010 777.

[27] C. Praveen, Y. W. Sagayaraj, P. T. Perumal, Tetrahedron Lett., 2009, 50,644 .

[28] C. Praveen, K. Karthikeyan, P. T. Perumal, Tetrahedron, 2009, 65, 9244.

[29] Y. P. Zhu, J. J. Yuan, Y. T. Li, M. Gao, L. P. Cao, J. Y. Ding, A. X. Wu, Synlett, 2011, 52.

[30] C. Praveen, A. Ayyanar, P. T. Perumal, Bioorg. Med. Chem. Lett., 2011, 21, 4170.

[31] H. Gasper, S. Santos, M. Carbone, A. S. Rodrigues, A. I. Rodrigues, M. J. Uriz, S. M. S. Feio, D. Melck, M. Humanes, M. Gavagnin, J. Nat. Prod., 2008, 71, 2049.

[32] H. Hikino, Y. Hikino, I. Yosioka, Chem. Pharm. Bull., 1964, 12, 755.

[33] R. C. Cambie, P. S. Rutledge, X. S. Yang, P. R. Bergquist, J. Nat. Prod., 1998, 61, 1416

[34] M. Gavagnin, E. Mollo, F. Castelluccio, A. Crispino, G. Cimino, J. Nat Prod., 2003, 66, 1517.

[35] H. Inouye, H. Matsumara, M. Kawasaski, K. Inoue, M. Tsukada, M. Tabata, Phytochemistry, 1981, 20, 1701.

[36] M. Kobayashi, A. Muroyama, H. Nakamura, J. Kobayashi, Y. Ohizumi, J. Pharmacol. Exp. Ther., 1991, 257, 90.

[37] H. Sakamoto, K. I. Furukawa, K. Matsunaga, H. Nakamura, Y. Ohizumi, Biochemistry, 1995, 34, 12570.

[38] W. L. Xiao, H. J. Zhu, Y. H. Shen, R. T. Li, S. H. Li, H. D. Sun, Y. T.
Zheng, R. R. Wang, Y. Lu, C. Wang, Q. T. Zheng, Org. Lett., 2005, 7, 2145.

[39] N. T. Patil, H. Wu, Y. Yamamoto, J. Org. Chem., 2005, 70, 4531.

[40] T. L. Yao, X. X. Zhang, R. C. Larock, J. Am. Chem. Soc., 2004, 126, 11164.

[41] S. Goncalves, A. Wagner, C. Mioskowski, R. Baati, Tetrahedron Lett., 2009, 50, 274.

[42] M. Yoshida, M. Al-Amin, K. Matsuda, K. Shishido, Tetrahedron Lett., 2008, $49,5021$.

[43] J. L. Zhang, H. G. Schmalz, Angew. Chem. Int. Ed., 2006, 45, 6704.

[44] A. S. K. Hashmi, L. Schwarz, J. H. Choi, T. M. Frost, Angew. Chem. Int. Ed., 2000, 39: 2285.

[45] C. H. Oh, V. R. Reddy, A. Kim, C. Y. Rhim, Tetrahedron Lett., 2006, $47,5307$.

[46] G. Mehta, N. S. Likhite, Tetrahedron Lett., 2008, 49, 7113.

[47] H-K Yim, Y. Liao, H. N. C. Wong, Tetrahedron, 2003, 59, 1877.

[48] A. Chakraborty, G. K. Kar, J. K. Ray, Tetrahedron, 1997, 53, 2989.

[49] K. Harada, Y. Tonoi, H. Kato, Y. Fukuyama, Tetrahedron Lett., 2002, $43,3829$.

[50] R. E. Patre, S. Gawas, S. Sen, P. S. Parameswaran, S. G. Tilve, Tetrahedron Lett., 2007, 48, 3517.

[51] J. S. Foot, A. T. Phillis, P. P. Sharp, A. C. Wills, M. G. Banwell, Tetrahedron Lett., 2006, 47, 6817.

[52] G. Blay, L. Cardona, B. Garca, J. R. Pedro, J. J. Snchez, J. Org. Chem., 1996, 61, 3815.

[53] M. Inoue, A. J. Frontier, S. J. Danishefsky, Angew. Chem. Int. Ed., 2000, 39, 761.

[54] M. Yoshida, M. Al-Amin, K. Shishido, Synthesis, 2009, 2454.

[55] A. Blanc, K. Tenbrink, J. M. Weibel, P. Pale, J. Org. Chem., 2009, 74, 5342.

[56] S. Borghèse, B. Louis, A. Blanc, P. Pale, Catal. Sci. Technol., 2011, 1, 981.

[57] C. Praveen, C. Iyyappan, P. T. Perumal, Tetrahedron Lett., 2010, 51, 4767.

[58] C. Praveen, C. Iyyappan, K. Girija, K. S. Kumar, P. T. Perumal, J. Chem. Sci., 2012, 124, 451.

[59] C. Praveen, A. Ayyanar, P. T. Perumal, Bioorg. Med. Chem. Lett., 
2011, 21, 4072 .

[60] C. Praveen, P. DheenKumar, D. Muralidharan, P. T. Perumal, Bioorg. Med. Chem. Lett., 2010, 20, 7292.

[61] K. Parthasarathy, C. Praveen, C. Balachandran, P. S. Kumar, S. Ignacimuthu, P. T. Perumal, Bioorg. Med. Chem. Lett., 2013, 23, 2708.

[62] K. Parthasarathy, C. Praveen, P. S. Kumar, C. Balachandran, P. T. Perumal, RSC Adv., 2015, 5, 15818.

[63] C. Praveen, S. Narendiran, P. Dheenkumar, P. T. Perumal, J. Chem. Sci., 2013, 125, 1543.

[64] C. R. Johnson, J. P. Adams, M. P. Braun, C. B. W. Senanayake, P. M. Wovkulich, M. R. Uskokoviḉ, Tetrahedron Lett., 1992, 33, 917.

[65] D. K. Mohapatra, B. Chatterjee, M. K. Gurjar, Tetrahedron Asymmetry, 2008, 19, 1568.

[66] C. K. Sha, S. J. Huang, Z. P. Zhan, J. Org. Chem., 2002, 67, 831.

[67] A. L. Germal, J. L. Luche, J. Am. Chem. Soc., 1981, 103, 5454.

[68] A. Mayasundari, D. G. J. Young, Tetrahedron Lett., 2001, 42, 203.

[69] K. Sonogashira, Y. Tohda, N. Hagihara, Tetrahedron Lett., 1975, 16 , 4467.

[70] R. Chinchilla, C. Nájera, Chem. Rev., 2007, 107, 874.

[71] E. Fillion, R. L. Beingessner, J. Org. Chem., 2003, 68, 9485.

[72] L. A. Paquette, J. Ezquerra, W. He, J. Org. Chem., 1995, 60, 1435.

[73] A. B. Neef, C. Schultz, Angew. Chem. Int. Ed., 2009, 48, 1498.

[74] A. E. Sadak, T. Arslan, N. Celebioglu, N. Saracoglu, Tetrahedron, 2010, 66, 3214
[75] C. B. Reese, A. Shaw, J. Chem. Soc. Perkin Trans. 1, 1975, 2422.

[76] G. Zeni, R. C. Larock, Chem. Rev., 2006, 106, 4644.

[77] T. W. Lyons, M. S. Sanford, Chem. Rev., 2010, 110, 1147.

[78] A. Bacchi, M. Costa, N. Della Cá, M. Fabbricatore, A. Fazio, B. Gabriele, C. Nasi, G. Salerno, Eur. J. Org. Chem., 2004, 574.

[79] A. Arcadi, S. Cacchi, M. D. Rosario, G. Fabrizi, F. Marinelli, J. Org. Chem., 1996, 61, 9280.

[80] B. Gabriele, G. Salerno, A. Fazio, R. Pittelli, Tetrahedron, 2003, 59, 6251.

[81] P. Peng, B. X. Tang, S. F. Pi, Y. Liang, J. H. Li, J. Org. Chem., 2009, 74, 3569.

[82] R. D. Stephens, C. E. Castro, J. Org. Chem., 1963, 28, 3313.

[83] E. Negishi, Acc. Chem. Res., 1982, 15, 340.

[84] E. Erdik, Tetrahedron, 1992, 48, 9577.

[85] M. Miyaura, A. Suzuki, Chem. Rev., 1995, 95, 2457.

[86] J. K. Stille, Angew. Chem. Int. Ed., 1986, 25, 508.

[87] H. A. Dieck, R. F. Heck, J. Am. Chem. Soc., 1974, 96, 1133.

[88] G. Dyker, E. Muth, A. S. K. Hashmi, L. Ding, Adv. Synth. Catal., 2003, $345,1247$.

[89] M. T. Reetz, K. Sommer, Eur. J. Org. Chem., 2003, 3485.

[90] J. A. Marshal, B. G. Shearer, S. L. Crooks, J. Org. Chem., 1987, 52, 1236.

[91] S. Kulyk, W. G. Dougherty Jr., W. S. Kassel, M. J. Zdilla, S. McN. Sieburth, Org. Lett., 2011, 13, 2180.

\title{
金催化的环化反应与钯催化的炔醇偶联/环化反应相结合用于 合成多取代呋喃衍生物: 串联反应的应用范围
}

\author{
Chandrasekar Praveen ${ }^{\text {a, }}$, Paramasivan T. Perumal ${ }^{b}$ \\ a 中央电化学研究所(科学与工业研究中心实验室)功能材料部, 卡莱科蒂630003, 印度 \\ ${ }^{\mathrm{b}}$ 中央皮革研究所(科学与工业研究中心实验室)有机化学部, 金奈600020, 印度
}

摘要: 开发了一种由金和钯催化 $\pi$-活化由炔醇合成呋喃衍生物的集成方法. 该合成策略是最显著的特点适用于带环辛基的底物, 其适用范围比之前报道的有很大扩展. 在Sonogashira反应条件下, 由相应底物可直接得到环辛基呋喃. Pd在这些反应中起到 2 个 重要作用: 底物发生偶联反应的关键催化剂; 通过 $\pi$-活化促进炔醇中间体成环反应. 该方法在一步合成3-硒呋喃反应中作用很突 出, 使通过偶联法进一步官能团化成为可能. 我们还将 $\mathrm{AuBr}_{3}$ 用于多米诺成环/C-H键活化反应和无环前体的成环反应. 本文结果 表明, 在该类成环反应中金和钯催化剂相辅相成.

关键词: 呋喃衍生物; 环化反应; 金催化; 钯催化; 一锅法; 串联反应

收稿日期: 2015-09-09. 接受日期: 2015-10-12. 出版日期: 2016-02-05.

*通讯联系人. 电话: +91-9677733808; 电子信箱: chandrasekar.praveen@gmail.com

基金来源: 印度科学技术部.

本文的英文电子版由Elsevier出版社在ScienceDirect上出版(http://www.sciencedirect.com/science/journal/18722067). 